DOI 10.4171/JEMS/192

Anar Akhmedov $\cdot$ Scott Baldridge $\cdot$ R. İnanç Baykur

· Paul Kirk · B. Doug Park

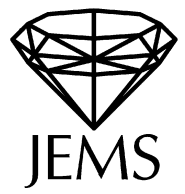

\title{
Simply connected minimal symplectic 4-manifolds with signature less than -1
}

Received December 9, 2007 and in revised form June 10, 2008

Abstract. For each pair $(e, \sigma)$ of integers satisfying $2 e+3 \sigma \geq 0, \sigma \leq-2$, and $e+\sigma \equiv 0$ (mod 4), with four exceptions, we construct a minimal, simply connected symplectic 4-manifold with Euler characteristic $e$ and signature $\sigma$. We also produce simply connected, minimal symplectic 4-manifolds with signature zero (resp. signature -1$)$ with Euler characteristic $4 k(r e s p .4 k+1)$ for all $k \geq 46$ (resp. $k \geq 49$ ).

Keywords. Symplectic topology, Luttinger surgery, fundamental group, 4-manifold

\section{Introduction}

In [6], a closed, simply connected, minimal symplectic 4-manifold with Euler characteristic 6 and signature -2 is constructed. This manifold contains a symplectic genus 2 surface with trivial normal bundle and simply connected complement and also contains two Lagrangian tori with special properties. In this article we use this manifold and apply standard constructions to fill out the part of the symplectic geography plane corresponding to signature less than -1 . Recall that Taubes proved ([35, 36, 37], also Li-Liu [21]) that minimal simply connected symplectic 4-manifolds satisfy $2 e+3 \sigma \geq 0$, where $e$ denotes the Euler characteristic and $\sigma$ the signature. Moreover, every symplectic 4-manifold satisfies $e+\sigma \equiv 0(\bmod 4)$.

Our main result is the following.

A. Akhmedov: School of Mathematics, University of Minnesota, Minneapolis, MN 55455, USA; e-mail: akhmedov@math.umn.edu

S. Baldridge: Department of Mathematics, Louisiana State University, Baton Rouge, LA 70817, USA; e-mail: sbaldrid@math.lsu.edu

R. İ. Baykur: Department of Mathematics, Brandeis University, Waltham, MA 02454, USA;

e-mail: baykur@brandeis.edu

P. Kirk: Mathematics Department, Indiana University, Bloomington, IN 47405, USA;

e-mail: pkirk@indiana.edu

B. D. Park: Department of Pure Mathematics, University of Waterloo, Waterloo, ON, N2L 3G1, Canada; e-mail: bdpark@math.uwaterloo.ca

Mathematics Subject Classification (2010): Primary 57R17; Secondary 57M05, 57R55 
Theorem A. Let $\sigma$ and e denote integers satisfying $2 e+3 \sigma \geq 0$ and $e+\sigma \equiv 0(\bmod 4)$. If, in addition,

$$
\sigma \leq-2
$$

then there exists a simply connected minimal symplectic 4-manifold with signature $\sigma$ and Euler characteristic e and odd intersection form, except possibly for $(\sigma, e)$ equal to $(-3,7),(-3,11),(-5,13)$, or $(-7,15)$.

In terms of $c_{1}^{2}$ and $\chi_{h}$, we construct symplectic manifolds realizing all pairs of integers satisfying $0 \leq c_{1}^{2} \leq 8 \chi_{h}-2$ except $\left(c_{1}^{2}, \chi_{h}\right)=(5,1),(13,2),(11,2)$, and $(9,2)$.

Using Freedman's theorem [13] and Taubes's results [36, 37] this theorem can be restated by saying that there exists a minimal symplectic manifold homeomorphic but not diffeomorphic to $m \mathbb{C P}^{2} \# n \overline{\mathbb{C P}}^{2}$ whenever $m+2 \leq n \leq 5 m+4$ and $m$ is odd, except possibly for $(m, n)=(1,4),(3,6),(3,8)$, or $(3,10)$. The existence of minimal symplectic 4-manifolds homeomorphic to $m \mathbb{C P}^{2} \# n \overline{\mathbb{C P}}^{2}$ for these four pairs remains an open problem (as far as we know).

The geography problem refers to the problem of determining which pairs $(\sigma, e)$ of integers arise as the signature and Euler characteristic of a 4-manifold in a certain class. The terminology was borrowed by topologists from algebraic geometers studying algebraic surfaces (see e.g. [7]). The motivation in 4-dimensional topology for studying the geography problem comes from Freedman's theorem [13] which shows that a simply connected smooth 4-manifold $M$ (with odd intersection form) is determined up to homeomorphism by the pair $(\sigma(M), e(M))$. The smooth geography problem has a long history: see [9]. The monograph [17] and the recent survey [12] contain a comprehensive list of references.

The study of the geography problem for symplectic 4-manifolds has been an area of active study in recent years. In his seminal paper [15], Gompf constructed simply connected symplectic 4-manifolds filling in a large part of the geography plane, most of which can be proven to be minimal by more recent techniques. J. Park explored the topic in a series of articles [26, 27], adressing minimality and uniqueness questions using SeibergWitten invariants. The articles [11, 19, 28, 33] have focused attention on the problem of constructing small simply connected symplectic manifolds. Recently, the approach introduced in [2] has spurred the discovery of new constructions of small simply connected symplectic manifolds; see [3, 4, 5, 6, 8, 30].

The methods in this article are based on inductive constructions to produce simply connected manifolds starting with a few basic non-simply connected models. Although there are some formal similarities between some of the fundamental group calculations carried out in this article and those in the articles [2, 3, 4, 5, 6, 8], there is an important difference, as we now explain.

In those articles, the mechanism used to kill fundamental groups comes down to establishing precise enough control over certain group presentations to conclude that all generators die. This is a subtle process which depends critically on properly identifying words in fundamental groups, since e.g. in a group, a pair of elements $x, y$ might commute but their conjugates $g x g^{-1}, h y h^{-1}$ need not. 
By contrast, the mechanism of the present paper is much softer. We use standard symplectic constructions pioneered by Gompf [15] and Luttinger [22] to kill a generator outright; subsequent generators are then killed by a simple argument. In particular, although we are explicit and careful in our fundamental group calculations in Theorem 1 . Lemma 16, and elsewhere, the reader will quickly understand that our results follow as easily if one only knows the statements up to conjugacy.

To illustrate this point, in the statement of Lemma 16 , the expressions for $\mu_{6}, m_{6}, \ell_{6}$ are long, but it is straightforward to see that, up to conjugacy, $\mu_{6}=\left[a_{1}, x_{2}\right], m_{6}=y_{2}$, $\ell_{6}=b_{1}^{-1}$. This less precise information is quite sufficient to prove the results of this article.

The construction is also suitable for filling out a large region of the geography plane starting with any given symplectic 4-manifold with given characteristic numbers and containing a square zero symplectic torus. For example, Theorem 23 roughly says that given a symplectic 4-manifold $X$, one can construct a new symplectic manifold $Y$ with the same fundamental group as $X$ and satisfying $c_{1}^{2}(Y)=c_{1}^{2}(X)+c$ and $\chi_{h}(Y)=\chi_{h}(X)+\chi$, for any $(c, \chi)$ in the cone $0 \leq c \leq 8 \chi-2$.

Since it is known how to produce manifolds with positive signature ([32]) we apply this result to a positive signature symplectic 4-manifold and prove the following.

Theorem B. For each integer $k \geq 45$, there exists a simply connected minimal symplectic 4-manifold $X_{2 k+1,2 k+1}$ with Euler characteristic $e=4 k+4$ and signature $\sigma=0$. For each integer $k \geq 49$, there exists a simply connected minimal symplectic 4-manifold $X_{2 k-1,2 k}$ with Euler characteristic $e=4 k+1$ and signature $\sigma=-1$.

All the manifolds we produce have odd intersection forms. Hence there remain four minimal simply connected symplectic odd 4-manifolds of signature less than or equal to -2 and 97 minimal simply connected symplectic odd 4-manifolds of non-positive signature yet to be constructed.

We finish this introduction with a brief description of the proofs. We start with three models, the minimal symplectic 4-manifolds $B, C, D$. These manifolds have Euler characteristic 6,8 , and 10 and signatures $-2,-4$, and -6 respectively. Each contains a disjoint pair of homologically independent Lagrangian tori $T_{1}$ and $T_{2}$ with nullhomotopic meridians and whose complement has fundamental group $\mathbb{Z} \oplus \mathbb{Z}$. Moreover, \pm 1 Luttinger surgery (see Section 2) along certain curves on one or both of these tori yields a minimal symplectic 4-manifold.

We then produce a family $B_{g}, g \in \mathbb{Z}$, of minimal symplectic 4-manifolds with Euler characteristic $6+4 g$ and signature -2 by taking a symplectic sum of $B$ with a minimal manifold constructed from Luttinger surgeries on a product of surfaces. This family $B_{g}$ again contains a pair of Lagrangian tori $T_{1}, T_{2}$ with the same properties as those in $B, C, D$.

Taking the symplectic sum of many copies of $B, B_{g}, C, D$ (and, if needed, the elliptic surfaces $E(k)$ ) along their tori and performing a +1 Luttinger surgery on each of the unused Lagrangian tori yields our even signature examples. Showing that the fundamental group vanishes is simple since the fundamental groups of $B, B_{g}, C, D$ and the homomorphisms induced by the inclusions of the tori are known. Usher's theorem [38] 
easily implies that the result is minimal. The manifolds $B, B_{g}, C, D$ contain -1 surfaces disjoint from the $T_{i}$ which survive to -1 surfaces in the symplectic sum and hence the result has an odd intersection form.

Producing odd signature manifolds follows the same general approach, but requires several small model manifolds with appropriate Lagrangian tori to use as seeds for the symplectic sums. The construction is not quite as clean as in the even signature case.

We construct a minimal symplectic 4 -manifold $P_{5,8}$ with fundamental group $\mathbb{Z}$, Euler characteristic 15 , and signature -3 . This and a few other known small manifolds with odd signature each contain a Lagrangian or symplectic torus appropriate for taking symplectic sums with many copies of $B, B_{g}, C$, and $D$. As in the even case, this produces minimal simply connected 4-manifolds of odd signature less than or equal to -5 .

The signature -3 examples are constructed by a separate argument, and a few small examples not covered by our general construction are culled from the literature (i.e. $(\sigma, e)=(-7,11),(-13,21),(-11,19),(-5,9))$ or constructed explicitly $((\sigma, e)=$ $(-5,17),(-7,19),(-9,21))$.

\section{Luttinger surgery}

Given any Lagrangian torus $T$ in a symplectic 4-manifold $M$, the Darboux-Weinstein theorem [23] implies that there is a parameterization of a tubular neighborhood $T^{2} \times D^{2}$ $\rightarrow \operatorname{nbd}(T) \subset M$ such that the image of $T^{2} \times\{d\}$ is Lagrangian for all $d \in D^{2}$. Choosing any point $d \neq 0$ in $D^{2}$ gives a push-off $F_{d}: T \rightarrow T^{2} \times\{d\} \subset M-T$ called the Lagrangian push-off or Lagrangian framing. Given any embedded curve $\gamma \subset T$, its image $F_{d}(\gamma)$ is called the Lagrangian push-off of $\gamma$.

Any curve isotopic to $\{t\} \times \partial D^{2} \subset \partial(\operatorname{nbd}(T))$ will be called a meridian of $T$ and typically denoted by $\mu_{T}$. In this article we will typically fix a pair of embedded curves on $T$ intersecting transversally in one point and denote the two Lagrangian push-offs by $m_{T}$ and $\ell_{T}$. The triple $\mu_{T}, m_{T}, \ell_{T}$ generate $H_{1}(\partial(\operatorname{nbd}(T)))$. Since the 3 -torus has abelian fundamental group we may choose a base point $t$ on $\partial(\operatorname{nbd}(T))$ and unambiguously refer to $\mu_{T}, m_{T}, \ell_{T} \in \pi_{1}(\partial(\operatorname{nbd}(T)), t)$.

The push-offs and meridian are used to specify coordinates for a Luttinger surgery. This is the process of removing a tubular neighborhood of $T$ in $M$ and regluing it so that the embedded curve representing $\mu_{T} m_{T}^{p} \ell_{T}^{q}$ bounds a disk for some pair of integers $p, q$. The resulting 4-manifold admits a symplectic structure whose symplectic form is unchanged away from a neighborhood of $T([1,22])$.

When the base point $x$ of $M$ is chosen off the boundary of the tubular neighborhood of $T$, the based loops $\mu_{T}, m_{T}$, and $\ell_{T}$ are to be joined to $x$ by the same path in $M-T$. These curves then define elements of $\pi_{1}(M-T, x)$. With $p, q$ as above, the 4-manifold resulting from Luttinger surgery on $M$ has fundamental group

$$
\pi_{1}(M-T, x) / N\left(\mu_{T} m_{T}^{p} \ell_{T}^{q}\right)
$$

where $N\left(\mu_{T} m_{T}^{p} \ell_{T}^{q}\right)$ denotes the normal subgroup generated by $\mu_{T} m_{T}^{p} \ell_{T}^{q}$.

We will only need the cases $(p, q)=( \pm 1,0)$ or $(0, \pm 1)$ in this article, i.e. \pm 1 Luttinger surgery along $m_{T}$ or $\ell_{T}$. 


\section{The fundamental group of the complement of tori in the product of surfaces}

Let $F$ be a genus $f$ surface, with $f \geq 2$. Choose a base point $h$ on $F$ and pairs $x_{i}, y_{i}$, $i=1, \ldots, f$, of circles forming a symplectic basis, with $x_{i}, y_{i}$ intersecting at $h_{i} \in F$. Choose paths $\alpha_{i}$ from $h$ to $h_{i}$, so that the loops

$$
\tilde{x}_{i}=\alpha_{i} x_{i} \alpha_{i}^{-1} \text { and } \quad \tilde{y}_{i}=\alpha_{i} y_{i} \alpha_{i}^{-1}
$$

generate $\pi_{1}(F, h)$. Let $Y_{i}$ be a circle parallel to $y_{i}$ which misses $\alpha_{i}$.

Let $G$ be a genus $g$ surface. Choose a base point $k$ on $G$, and $g$ pairs $a_{1}, b_{1}, \ldots, a_{g}, b_{g}$ of circles forming a symplectic basis, with $a_{i}, b_{i}$ intersecting at $k_{i}$. Choose paths $\beta_{i}$ from $k$ to $k_{i}$, so that the loops

$$
\tilde{a}_{i}=\beta_{i} a_{i} \beta_{i}^{-1} \quad \text { and } \quad \tilde{b}_{i}=\beta_{i} b_{i} \beta_{i}^{-1}
$$

generate $\pi_{1}(G, k)$. Choose parallel copies $A_{i}$ of $a_{i}$ and $B_{i}$ of $b_{i}$ which miss the paths $\beta_{i}$. In Figure 1 we illustrate the notation when $f=2$ and $g=3$.
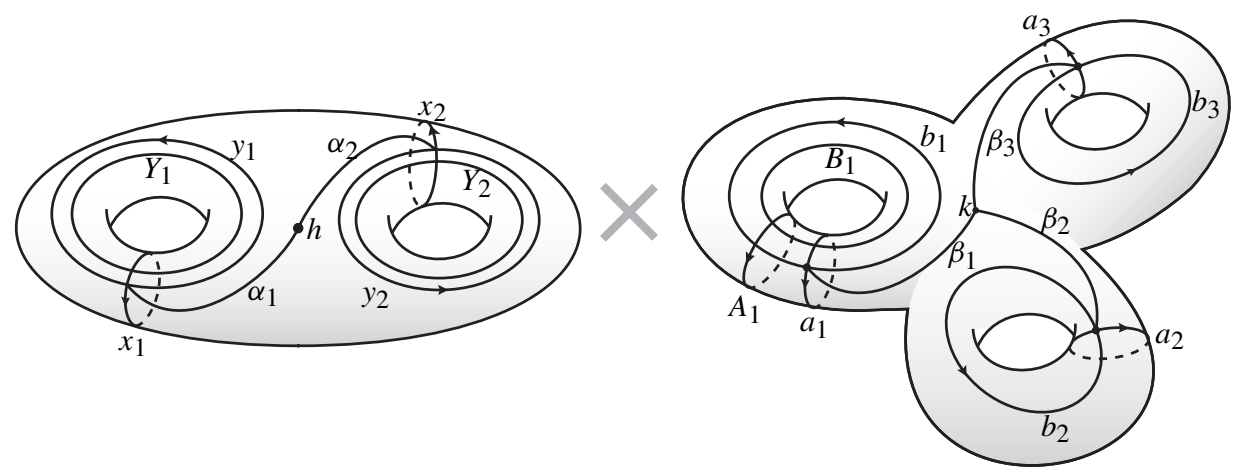

Fig. 1. The surface $F \times G$.

The product $F \times G$ contains the union of the two symplectic surfaces $F \times\{k\} \cup\{h\} \times G$ meeting at $(h, k)$. There is an identification $\pi_{1}(F \times G,(h, k))=\pi_{1}(F, h) \times \pi_{1}(G, k)$ which associates the loop $\tilde{x}_{i} \times\{k\}$ to $\left(\tilde{x}_{i}, 1\right), \tilde{y}_{i} \times\{k\}$ to $\left(\tilde{y}_{i}, 1\right),\{h\} \times \tilde{a}_{i}$ to $\left(1, \tilde{a}_{i}\right)$ and $\{h\} \times$ $\tilde{b}_{i}$ to $\left(1, \tilde{b}_{i}\right)$. In other words, the homomorphisms induced by the inclusions $F \times\{k\} \subset$ $F \times G$ and $\{h\} \times G \subset F \times G$ present $\pi_{1}(F \times G,(h, k))$ as the product of $\pi_{1}(F, h)$ and $\pi_{1}(G, k)$.

When there is no chance of confusion we denote the $2 f+2 g$ loops $\tilde{x}_{i} \times\{k\}, \tilde{y}_{i} \times\{k\}$, $\{h\} \times \tilde{a}_{i},\{h\} \times \tilde{b}_{i}$ simply by $\tilde{x}_{i}, \tilde{y}_{i}, \tilde{a}_{i}, \tilde{b}_{i}$. These are loops in $F \times G$ based at $(h, k)$.

The product $F \times G$ contains $2 g$ Lagrangian tori

$$
Y_{1} \times A_{j}, \quad Y_{2} \times B_{j}, \quad j=1, \ldots, g .
$$

These $2 g$ tori are pairwise disjoint and miss $(F \times\{k\}) \cup(\{h\} \times G)$.

Let $N$ denote a tubular neighborhood of the union of these $2 g$ tori:

$$
N=\operatorname{nbd}\left(\left(\bigcup_{j} Y_{1} \times A_{j}\right) \cup\left(\bigcup_{j} Y_{2} \times B_{j}\right)\right) \subset F \times G .
$$

The loops $\tilde{x}_{i}, \tilde{y}_{i}, \tilde{a}_{i}, \tilde{b}_{i}$ are loops in $F \times G-N$ based at $(h, k)$. 
Typically, removing a surface from a 4-manifold increases the number of generators of the fundamental group, but since these tori respect the product structure one can prove the following theorem.

Theorem 1. The $2 f+2 g$ loops $\tilde{x}_{1}, \tilde{y}_{1}, \ldots, \tilde{x}_{f}, \tilde{y}_{f}, \tilde{a}_{1}, \tilde{b}_{1}, \ldots, \tilde{a}_{g}, \tilde{b}_{g}$ generate $\pi_{1}(F \times G$ $-N,(h, k))$. There are paths $d_{j}:[0,1] \rightarrow F \times G-N$ from $(h, k)$ to the boundary of the tubular neighborhood of $Y_{1} \times A_{j}$ and $e_{j}:[0,1] \rightarrow F \times G-N$ from $(h, k)$ to the boundary of the tubular neighborhood of $Y_{2} \times B_{j}$ so that with respect to these paths, the meridian and two Lagrangian push-offs of $Y_{1} \times A_{j}$ are homotopic in $F \times G-N$ rel endpoint to

$$
\mu_{Y_{1} \times A_{j}}=\left[\tilde{x}_{1}, \tilde{b}_{j}\right], \quad m_{Y_{1} \times A_{j}}=\tilde{y}_{1}, \quad \ell_{Y_{1} \times A_{j}}=\tilde{a}_{j},
$$

and the meridian and two Lagrangian push-offs of $Y_{2} \times B_{j}$ are homotopic in $F \times G-N$ rel endpoint to

$$
\mu_{Y_{2} \times B_{j}}=\left[\tilde{x}_{2}, \tilde{a}_{j}\right], \quad m_{Y_{2} \times B_{j}}=\tilde{y}_{2}, \quad \ell_{Y_{2} \times B_{j}}=\tilde{b}_{j} .
$$

Proof. Before we start the proof, we give an indication of how it will proceed. Note that $\bigcup_{j}\left(Y_{1} \times A_{j}\right)=Y_{1} \times \bigcup_{j} A_{j}$ lies on $Y_{1} \times G$ and that $Y_{2} \times \bigcup_{j} B_{j}$ lies on $Y_{2} \times G$. Thus $F \times G-N$ can be constructed by cutting $F \times G$ along the hypersurface $\left(Y_{1} \cup Y_{2}\right) \times G$, and then regluing the two copies of $Y_{1} \times G$ only along the complement of a neighborhood of the $A_{j}$, and regluing the two copies of $Y_{2} \times G$ only along the complement of a neighborhood of the $B_{j}$. However, in order to use the Seifert-Van Kampen theorem, the subsets and their intersection in a decomposition are required to be connected, and so we need to modify the decomposition slightly.

Let $P_{1}$ be the annulus in $F$ bounded by $y_{1}$ and $Y_{1}$. Similarly let $P_{2}$ denote the annulus in $F$ bounded by $y_{2}$ and $Y_{2}$. Let $\alpha$ denote the arc $\left(\alpha_{1} \cup \alpha_{2}\right) \times\{k\}$. Let $\gamma_{1}$ denote the arc $\left(x_{1} \cap P_{1}\right) \times\{k\}$; it spans the two circles $y_{1}$ and $Y_{1}$. Similarly let $\gamma_{2}$ denote the arc $\left(x_{2} \cap P_{2}\right) \times\{k\}$. See Figure 2. Set

$$
S_{1}=\left(P_{1} \times G\right) \cup \alpha \cup\left(P_{2} \times G\right), \quad S_{2}=\left(\gamma_{1} \cup \gamma_{2}\right) \cup\left(\left(F-\operatorname{int}\left(P_{1} \cup P_{2}\right)\right) \times G\right) .
$$

Then in $F \times G, S_{1} \cap S_{2}$ is the union of four copies of $S^{1} \times G$ together with three arcs which connect the four components. In particular, $S_{1}, S_{2}$ and $S_{1} \cap S_{2}$ are connected and contain the base point $(h, k)$.

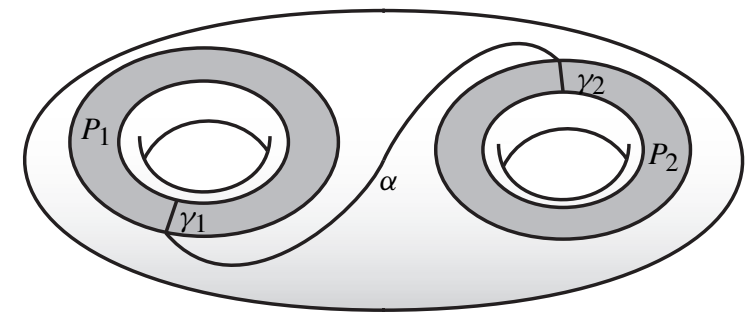

Fig. 2 
Let $G_{A}=G-\operatorname{nbd}\left(\bigcup_{j} A_{j}\right)$ denote the complement of an open tubular neighborhood of the $A_{j}$ in $G$. Since the $A_{j}$ do not disconnect $G, G_{A}$ is path connected. Similarly let $G_{B}=G-\operatorname{nbd}\left(\bigcup_{j} B_{j}\right)$ denote the complement of an open tubular neighborhood of the $B_{j}$ in $G$.

To construct $F \times G-N$ we form the identification space

$$
F \times G-N=S_{1} \sqcup S_{2} / \sim
$$

by identifying $(f, s) \in S_{1}$ with its corresponding point $\left(f^{\prime}, s^{\prime}\right)$ in $S_{2}$ except if $f \in Y_{1}$ and $s \in \operatorname{nbd}\left(A_{j}\right)$ or $f \in Y_{2}$ and $s \in \operatorname{nbd}\left(B_{j}\right)$. In other words, along $Y_{1} \times G$ we identify only the two copies of $Y_{1} \times G_{A}$ and along $Y_{2} \times G$ we identify only the two copies of $Y_{2} \times G_{B}$.

Hence we have exhibited $F \times G-N$ as the union of $S_{1}$ and $S_{2}$ with connected intersection

$$
S_{1} \cap S_{2}=\left(Y_{1} \times G_{A}\right) \cup \gamma_{1} \cup\left(y_{1} \times G\right) \cup \alpha \cup\left(y_{2} \times G\right) \cup \gamma_{2} \cup\left(Y_{2} \times G_{B}\right) .
$$

It is easy to see that $\pi_{1}\left(S_{1} \cap S_{2},(h, k)\right) \rightarrow \pi_{1}\left(S_{1},(h, k)\right)$ is surjective. Indeed, one can use the product parameter in the annuli $P_{1}$ and $P_{2}$ to define a deformation retraction (fixing $\alpha$ and hence also $(h, k))$ of $S_{1}$ to the subset $\left(y_{1} \times G\right) \cup \alpha \cup\left(y_{2} \times G\right)$ of $S_{1} \cap S_{2}$.

The Seifert-Van Kampen theorem applies and implies that there is a surjection

$$
\pi_{1}\left(S_{2},(h, k)\right) \rightarrow \pi_{1}(F \times G-N,(h, k))
$$

induced by inclusion.

We will show that the image of $\pi_{1}\left(S_{2},(h, k)\right) \rightarrow \pi_{1}(F \times G-N,(h, k))$ is generated by the loops $\tilde{x}_{1}, \tilde{y}_{1}, \tilde{x}_{2}, \tilde{y}_{2}, \ldots, \tilde{x}_{f}, \tilde{y}_{f}, \tilde{a}_{1}, \tilde{b}_{1}, \ldots, \tilde{a}_{g}, \tilde{b}_{g}$. Notice that all these loops are contained in $S_{2}$.

We find generators for $\pi_{1}\left(S_{2},(h, k)\right)$. This is again a straightforward application of the Seifert-Van Kampen theorem, as we will now show.

Since the arcs $\gamma_{i}$ are just segments that lie on $\tilde{x}_{i}$ (and the rest of the loops $\tilde{x}_{i}$ lie in $S_{2}$ ), we can decompose $S_{2}$ as

$$
S_{2}=\left(\tilde{x}_{1} \cup \tilde{x}_{2}\right) \cup\left(\left(F-\operatorname{int}\left(P_{1} \cup P_{2}\right)\right) \times G\right) .
$$

The intersection of the two pieces in this decomposition is the (contractible) set $\tilde{x}_{1} \cup \tilde{x}_{2}-$ $\left(\gamma_{1} \cup \gamma_{2}\right)$.

Hence $\pi_{1}\left(S_{2},(h, k)\right)$ is generated by $\tilde{x}_{1}, \tilde{x}_{2}$ and any set of generators of

$$
\pi_{1}\left(\left(F-\operatorname{int}\left(P_{1} \cup P_{2}\right)\right) \times G,(h, k)\right)=\pi_{1}\left(F-\operatorname{int}\left(P_{1} \cup P_{2}\right), h\right) \times \pi_{1}(G, k) .
$$

The loops $\tilde{a}_{i}, \tilde{b}_{i}$ generate $\pi_{1}(G, k)$. The space $F-\operatorname{int}\left(P_{1} \cup P_{2}\right)$ is a 4-punctured genus $f-2$ surface. Its fundamental group is generated by $\tilde{y}_{1}, \tilde{y}_{2}, \tilde{x}_{3}, \tilde{y}_{3}, \ldots, \tilde{x}_{f}, \tilde{y}_{f}$ and one other loop $\tau$ based at $h$ which is obtained by traveling from the base point to a point on the boundary component $Y_{1}$, following $Y_{1}$, then returning to the base point.

We have shown that the loops $\tilde{x}_{1}, \tilde{y}_{1}, \tilde{x}_{2}, \tilde{y}_{2}, \tilde{x}_{3}, \tilde{y}_{3}, \ldots, \tilde{x}_{f}, \tilde{y}_{f}, \tilde{a}_{1}, \tilde{b}_{1}, \ldots, \tilde{a}_{g}, \tilde{b}_{g}$, and $\tau \times\{k\}$ generate $\pi_{1}\left(S_{2},(h, k)\right)$. Hence, considered as loops in $F \times G-N$, they generate $\pi_{1}(F \times G-N,(h, k))$. We need only show that the generator $\tau \times\{k\}$ is not 
needed. But this is obvious since $\tilde{x}_{1}, \tilde{y}_{1}, \ldots, \tilde{x}_{f}, \tilde{y}_{f}$ and $\tau \times\{k\}$ all lie on the surface $F \times\{k\} \subset F \times G-N$, and $\tilde{x}_{1}, \tilde{y}_{1}, \ldots, \tilde{x}_{f}, \tilde{y}_{f}$ generate $\pi_{1}(F \times\{k\},(h, k))$.

We next turn to the problem of expressing the meridians and Lagrangian push-offs of the generators of the Lagrangian tori $Y_{1} \times A_{j}, Y_{2} \times B_{j}$ in terms of the loops $\tilde{x}_{1}, \tilde{y}_{1}, \ldots, \tilde{x}_{f}$, $\tilde{y}_{f}, \tilde{a}_{1}, \tilde{b}_{1}, \ldots, \tilde{a}_{g}, \tilde{b}_{g}$. We do this for $Y_{1} \times A_{1}$. Symmetric arguments provide the analogous calculations for the rest.

In Figure 1, denote by $h_{1}$ the intersection of $x_{1}$ and $y_{1}$ (i.e. the endpoint of $\alpha_{1}$ ), and by $k_{1}$ the intersection of $a_{1}$ and $b_{1}$. Then the point $\left(h_{1}, k_{1}\right)$ lies on the boundary of a tubular neighborhood of $Y_{1} \times A_{1}$.

Since we take the product symplectic form on $F \times G$, referring to Figure 1 one sees that the loops $y_{1} \times\left\{k_{1}\right\}$ and $\left\{h_{1}\right\} \times a_{1}$ are Lagrangian push-offs of two generators of $\pi_{1}\left(Y_{1} \times A_{1}\right)$ to the boundary of the tubular neighborhood of $Y_{1} \times A_{1}$.

There is a map of a square into $F \times G-N$ given by $\alpha_{1} \times \beta_{1}$ :

$$
\alpha_{1} \times \beta_{1}:[0,1] \times[0,1] \rightarrow F \times G-N .
$$

The point $(0,0)$ is mapped to the base point $(h, k)$ of $F \times G-N$, and $(1,1)$ is mapped to $\left(h_{1}, k_{1}\right)$. Thus the diagonal path $d(t)=\left(\alpha_{1}(t), \beta_{1}(t)\right)$ connects the base point to the boundary of the tubular neighborhood of $Y_{1} \times A_{1}$. So

Conjugating by $d$ expresses the Lagrangian push-offs as based curves in $F \times G-N$.

$$
m_{Y_{1} \times A_{1}}=d *\left(y_{1} \times\left\{k_{1}\right\}\right) * d^{-1} \quad \text { and } \quad \ell_{Y_{1} \times A_{1}}=d *\left(\left\{h_{1}\right\} \times a_{1}\right) * d^{-1} .
$$

But $m_{Y_{1} \times A_{1}}$ is homotopic rel basepoint in $F \times G-N$ to $\tilde{y}_{1}$. An explicit homotopy is given by the formula

$$
(s, t) \mapsto \begin{cases}\left(\alpha_{1}(3 t), \beta_{1}((1-s) 3 t)\right) & \text { if } 0 \leq t \leq 1 / 3, \\ \left(y_{1}(3 t-1), \beta_{1}(1-s)\right) & \text { if } 1 / 3 \leq t \leq 2 / 3, \\ \left(\alpha_{1}(3-3 t), \beta_{1}((1-s)(3-3 t))\right) & \text { if } 2 / 3 \leq t \leq 1 .\end{cases}
$$

A similar homotopy, but exchanging the roles of $\alpha_{1}$ and $\beta_{1}$, establishes that $\ell_{Y_{1} \times A_{1}}$ is homotopic rel basepoint in $F \times G-N$ to $\tilde{a}_{1}$. (These homotopies clearly miss all the other $Y_{1} \times A_{j}$ and $Y_{2} \times B_{j}$.)

It remains to calculate the meridian of $Y_{1} \times A_{1}$. For this, consider the map $x_{1} \times b_{1}$ : $[0,1] \times[0,1] \rightarrow F \times G$. This has image a torus intersecting $Y_{1} \times A_{1}$ transversally in one point (near the point $\left(x_{1}(.9), b_{1}(.9)\right)$, as one sees from Figure 1). Since

$$
\left(h_{1}, k_{1}\right)=\left(x_{1} \times b_{1}\right)(0,0)=\left(x_{1} \times b_{1}\right)(0,1)=\left(x_{1} \times b_{1}\right)(1,0)=\left(x_{1} \times b_{1}\right)(1,1),
$$

by conjugating the path that follows the boundary of this square by the path $d$ from the base point $(h, k)$ to $\left(h_{1}, k_{1}\right)$, we see that the meridian $\mu_{Y_{1} \times A_{1}}$ is given by the composite

$$
\mu_{Y_{1} \times A_{1}}=d *\left(x_{1} \times\left\{k_{1}\right\}\right) *\left(\left\{h_{1}\right\} \times b_{1}\right) *\left(x_{1} \times\left\{k_{1}\right\}\right)^{-1} *\left(\left\{h_{1}\right\} \times b_{1}\right)^{-1} * d^{-1} .
$$


Now $d *\left(x_{1} \times\left\{k_{1}\right\}\right) * d^{-1}$ is homotopic rel basepoint to $\tilde{x}_{1}$ in $F \times G-N$ by the same argument given above. The key observation is that $\beta_{1}$ misses $A_{j}$ and $B_{j}$ for all $j$. Similarly $d *\left(\left\{h_{1}\right\} \times b_{1}\right) * d^{-1}$ is homotopic rel basepoint to $\tilde{b}_{1}$ in $F \times G-N$. Thus

$$
\mu_{Y_{1} \times A_{1}} \sim \tilde{x}_{1} * \tilde{b}_{1} * \tilde{x}_{1}^{-1} * \tilde{b}_{1}^{-1}=\left[\tilde{x}_{1}, \tilde{b}_{1}\right]
$$

Similar calculations establish all other assertions.

\section{Telescoping triples and symplectic sums}

Our construction of symplectic 4-manifolds which fill large regions in the geography plane is based on using telescoping symplectic sums along symplectic tori as well as Luttinger surgeries. The basic models in our constructions have a convenient property preserved under appropriate symplectic sum, and so we formalize the property in the following definition.

Definition 2. An ordered triple $\left(X, T_{1}, T_{2}\right)$ where $X$ is a symplectic 4-manifold and $T_{1}, T_{2}$ are disjointly embedded Lagrangian tori is called a telescoping triple if

(1) The tori $T_{1}, T_{2}$ span a 2-dimensional subspace of $H_{2}(X ; \mathbb{R})$.

(2) $\pi_{1}(X) \cong \mathbb{Z}^{2}$ and the inclusion induces an isomorphism $\pi_{1}\left(X-\left(T_{1} \cup T_{2}\right)\right) \rightarrow \pi_{1}(X)$ (in particular the meridians of the $T_{i}$ are trivial in $\pi_{1}\left(X-\left(T_{1} \cup T_{2}\right)\right)$ ).

(3) The image of the homomorphism induced by inclusion $\pi_{1}\left(T_{1}\right) \rightarrow \pi_{1}(X)$ is a summand $\mathbb{Z} \subset \pi_{1}(X)$.

(4) The homomorphism induced by inclusion $\pi_{1}\left(T_{2}\right) \rightarrow \pi_{1}(X)$ is an isomorphism.

If $X$ is minimal we call $\left(X, T_{1}, T_{2}\right)$ a minimal telescoping triple.

Note that the order of $\left(T_{1}, T_{2}\right)$ matters in this definition. Notice also that since the meridians $\mu_{T_{1}}, \mu_{T_{2}} \in \pi_{1}\left(X-\left(T_{1} \cup T_{2}\right)\right)$ are trivial and the relevant fundamental groups are abelian, the push-off of an oriented loop $\gamma \subset T_{i}$ into $X-\left(T_{1} \cup T_{2}\right)$ with respect to any framing of the normal bundle of $T_{i}$ (e.g. the Lagrangian framing) represents a well defined element of $\pi_{1}\left(X-\left(T_{1} \cup T_{2}\right)\right)$, independent of the choice of framing (and basing).

The definition of a telescoping triple includes the hypothesis that the Lagrangian tori $T_{1}$ and $T_{2}$ are linearly independent in $H_{2}(X ; \mathbb{R})$. This implies $([15])$ that the symplectic form on $X$ can be slightly perturbed so that one of the $T_{i}$ remains Lagrangian while the other becomes symplectic. It can also be perturbed so that both become symplectic. Moreover, if $F$ is a symplectic surface in $X$ disjoint from $T_{1}$ and $T_{2}$, the perturbed symplectic form can be chosen so that $F$ remains symplectic.

Recall that the symplectic sum ([15]) of two symplectic 4-manifolds $X$ and $X^{\prime}$ along genus $g$ symplectic surfaces $F \subset X$ and $F^{\prime} \subset X$ of opposite self-intersection is a symplectic 4-manifold described topologically as the union

$$
X \#_{F, F^{\prime}} X^{\prime}=(X-\operatorname{nbd}(F)) \cup\left(X^{\prime}-\operatorname{nbd}\left(F^{\prime}\right)\right)
$$

where the boundaries of the tubular neighborhoods are identified by a fiber-preserving diffeomorphism of the corresponding circle bundles. When the surfaces are clear from context we write $X \#_{S} X^{\prime}$. 
Proposition 3. Let $\left(X, T_{1}, T_{2}\right)$ and $\left(X^{\prime}, T_{1}^{\prime}, T_{2}^{\prime}\right)$ be two telescoping triples. Then for an appropriate gluing map the triple

$$
\left(X \#_{T_{2}, T_{1}^{\prime}} X^{\prime}, T_{1}, T_{2}^{\prime}\right)
$$

is again a telescoping triple. The Euler characteristic and signature of $X \#_{T_{2}, T_{1}^{\prime}} X^{\prime}$ are given by $e(X)+e\left(X^{\prime}\right)$ and $\sigma(X)+\sigma\left(X^{\prime}\right)$.

Proof. Let $i_{j}: \pi_{1}\left(T_{j}\right) \rightarrow \pi_{1}(X)$ be the homomorphisms induced by inclusion for $j=$ 1,2. Choose $x_{1}, y_{1} \in \pi_{1}\left(T_{1}\right)$ so that $x_{1}$ spans the kernel of $i_{1}$ and $i_{1}\left(y_{1}\right)$ spans the image of $i_{1}$. Denote $i_{1}\left(y_{1}\right)$ by $t$ and choose $s \in \pi_{1}(X)$ so that $s, t$ forms a basis of $\pi_{1}(X)$. Then choose generators $x_{2}, y_{2}$ for $\pi_{1}\left(T_{2}\right)$ so that $i_{2}\left(x_{2}\right)=s$ and $i_{2}\left(y_{2}\right)=t$. Thus the inclusions induce

$$
x_{1} \mapsto 1, \quad y_{1} \mapsto t, \quad x_{2} \mapsto s, \quad y_{2} \mapsto t .
$$

Similarly, construct generators $x_{1}^{\prime}, y_{1}^{\prime}$ for $\pi_{1}\left(T_{1}^{\prime}\right), x_{2}^{\prime}, y_{2}^{\prime}$ for $\pi_{1}\left(T_{2}^{\prime}\right)$ and $s^{\prime}, t^{\prime}$ for $\pi_{1}\left(X^{\prime}\right)$.

The inclusion induces an isomorphism $\pi_{1}\left(X-\left(T_{1} \cup T_{2}\right)\right) \rightarrow \pi_{1}(X)$ and the boundary of the tubular neighborhood of $T_{1}$ (resp. $T_{2}$ ) is a 3-torus whose fundamental group is spanned by $x_{1}, y_{1}, \mu_{T_{1}}$ (resp. $x_{2}, y_{2}, \mu_{T_{2}}$ ) (for definiteness use the Lagrangian framing to push the $x_{i}, y_{i}$ into the boundary of the tubular neighborhood). Similar assertions hold for $\left(X^{\prime}, T_{1}^{\prime}, T_{2}^{\prime}\right)$. The symplectic sum of $X$ and $X^{\prime}$ along the surfaces $T_{2} \subset X$ and $T_{1}^{\prime} \subset X^{\prime}$ can be formed so that the ordered triple $\left(x_{2}, y_{2}, \mu_{2}\right)$ is sent to $\left(x_{1}^{\prime}, y_{1}^{\prime}, \mu_{1}^{\prime}\right)$ by the gluing diffeomorphism (perhaps after a change of orientation on some of the loops to ensure that the gluing diffeomorphism is orientation preserving).

The Seifert-Van Kampen theorem and the fact that all meridians are trivial imply that

$$
\pi_{1}\left(X \#_{T_{2}, T_{1}^{\prime}} X^{\prime}\right)=\left\langle s, t, s^{\prime}, t^{\prime} \mid[s, t],\left[s^{\prime}, t^{\prime}\right], s, t\left(t^{\prime}\right)^{-1}\right\rangle=\mathbb{Z} s^{\prime} \oplus \mathbb{Z} t^{\prime} .
$$

The inclusion $T_{1} \subset X \#_{T_{2}, T_{1}^{\prime}} X^{\prime}$ induces $x_{1} \mapsto 1, y_{1} \mapsto t^{\prime}$. The inclusion $T_{2}^{\prime} \subset X \#_{T_{2}, T_{1}^{\prime}} X^{\prime}$ induces $x_{2}^{\prime} \mapsto s^{\prime}, y_{2}^{\prime} \mapsto t^{\prime}$. Hence $\left(X \#_{T_{2}, T_{1}^{\prime}} X^{\prime}, T_{1}, T_{2}^{\prime}\right)$ is indeed a telescoping triple.

The assertions about the Euler characteristic and signature are clear.

Since the meridians of the Lagrangian tori are trivial in a telescoping triple, one immediately deduces the following.

Proposition 4. Let $\left(X, T_{1}, T_{2}\right)$ be a telescoping triple. Let $\ell_{T_{1}}$ be a Lagrangian push-off of a curve on $T_{1}$ and $m_{T_{2}}$ the Lagrangian push-off of a curve on $T_{2}$ so that $\ell_{T_{1}}$ and $m_{T_{2}}$ generate $\pi_{1}(X)$. Then the symplectic 4-manifold obtained by performing +1 Luttinger surgery on $T_{1}$ along $\ell_{T_{1}}$ and +1 surgery on $T_{2}$ along $m_{T_{2}}$ is simply connected.

We will make frequent use of the following two results. The first is a criterion given by Usher [38] to determine when a symplectic sum is minimal. The second is a useful result of T.-J. Li which we will use to verify that the hypotheses in Usher's theorem hold in certain contexts.

Theorem 5 (Usher). Let $Z=X_{1} \#_{F_{1}, F_{2}} X_{2}$ denote the symplectic sum of $X_{1}$ and $X_{2}$ along symplectic surfaces $F_{i}$ of positive genus $g$. Then: 
(i) If either $X_{1}-F_{1}$ or $X_{2}-F_{2}$ contains an embedded symplectic sphere of square -1 , then $Z$ is not minimal.

(ii) If one of the summands $X_{i}$ (for definiteness, say $X_{1}$ ) admits the structure of an $S^{2}$ bundle over a surface of genus $g$ such that $F_{1}$ is a section of this fiber bundle, then $Z$ is minimal if and only if $X_{2}$ is minimal.

(iii) In all other cases, $Z$ is minimal.

Corollary 3 of T.-J. Li's article [20] provides a useful method to eliminate the first two cases of Usher's theorem in some contexts.

Theorem 6 (Li). Let $M$ be a symplectic 4-manifold which is neither rational nor ruled. Then every smoothly embedded - 1 sphere is homologous to a symplectic -1 curve up to sign. If $M$ is the blowup of a minimal symplectic 4 -manifold with $E_{1}, \ldots, E_{n}$ represented by exceptional curves, then the $E_{i}$ are the only classes represented by a smoothly embedded -1 sphere, hence any orientation preserving diffeomorphism maps $E_{i}$ to some $\pm E_{j}$.

\section{The model even signature manifolds}

We will set up an inductive argument by constructing telescoping symplectic sums starting with several basic telescoping triples. Proposition 4 then applies to produce simply connected 4-manifolds.

To begin with, in [6. Theorem 20], a minimal telescoping triple $\left(B, T_{1}, T_{2}\right)$ is constructed ( $B$ is denoted $B_{1}$ in that article) so that $B$ contains a genus 2 surface $F$ with trivial normal bundle, and a geometrically dual symplectic -1 torus $H_{1}$. The tori $T_{1}, T_{2}$ miss $F \cup H_{1}$. Moreover, $\left(B-F, T_{1}, T_{2}\right)$ is also a telescoping triple. These facts follow immediately from the following theorem, which summarizes the assertions established in [6].

Theorem 7. There exists a minimal symplectic 4-manifold B containing a pair of homologically essential Lagrangian tori $T_{1}$ and $T_{2}$ and a square zero symplectic genus 2 surface $F$ so that $T_{1}, T_{2}$, and $F$ are pairwise disjoint, $e(B)=6$ and $\sigma(B)=-2$, and:

(1) $\pi_{1}\left(B-\left(F \cup T_{1} \cup T_{2}\right)\right)=\mathbb{Z}^{2}$, generated by $t_{1}$ and $t_{2}$.

(2) The inclusion $B-\left(F \cup T_{1} \cup T_{2}\right) \subset B$ induces an isomorphism on fundamental groups. In particular, the meridians $\mu_{F}, \mu_{T_{1}}, \mu_{T_{2}}$ all vanish in $\pi_{1}\left(B-\left(F \cup T_{1} \cup T_{2}\right)\right)$.

(3) The Lagrangian push-offs $m_{T_{1}}, \ell_{T_{1}}$ of $\pi_{1}\left(T_{1}\right)$ are sent to 1 and $t_{2}$ respectively in the fundamental group of $B-\left(F \cup T_{1} \cup T_{2}\right)$.

(4) The Lagrangian push-offs $m_{T_{2}}, \ell_{T_{2}}$ of $\pi_{1}\left(T_{2}\right)$ are sent to $t_{1}$ and $t_{2}$ respectively in the fundamental group of $B-\left(F \cup T_{1} \cup T_{2}\right)$.

(5) The push-off $F \subset B-\left(F \cup T_{1} \cup T_{2}\right)$ takes the first three generators of a standard symplectic generating set $\left\{a_{1}, b_{1}, a_{2}, b_{2}\right\}$ for $\pi_{1}(F)$ to 1 and the last element to $t_{2}$.

(6) There exists a symplectic torus $H_{1} \subset B$ which intersects $F$ transversally once, which has square -1 , and the homomorphism $\pi_{1}\left(H_{1}\right) \rightarrow \pi_{1}(B)$ takes the first generator to 1 and the second to $t_{1}$. Moreover, $H_{1}$ is disjoint from $T_{1}$ and $T_{2}$ (see [6, Proposition 12, Theorem 20]). 
The following is a restatement of [6, Theorem 13]. We state it formally since we will have frequent need of it.

Corollary 8. The symplectic 4-manifold $X_{1,3}$ obtained from $B$ by +1 Luttinger surgery on $T_{1}$ along $\ell_{T_{1}}$ and +1 Luttinger surgery on $T_{2}$ along $m_{T_{2}}$ is a minimal symplectic 4-manifold homeomorphic to $\mathbb{C P}^{2} \# 3 \overline{\mathbb{C P}}^{2}$. It contains a genus 2 symplectic surface of square zero with simply connected complement and a symplectic torus $H_{1}$ of square -1 intersecting $F$ transversally and positively in one point.

Corollary 9. For each $g \geq 0$ there exists a minimal telescoping triple $\left(B_{g}, T_{1}, T_{2}\right)$ satisfying $e\left(B_{g}\right)=6+4 g$ and $\sigma\left(B_{g}\right)=-2$ and containing a square -1 genus $g+1$ surface disjoint from $T_{1} \cup T_{2}$.

Proof. To avoid confusing notation, during this proof we denote the symplectic genus 2 surface in $B$ of Theorem 7 by $F_{B}$.

Take the product $F \times G$ of a genus 2 surface $F$ and a genus $g$ surface $G$, as in Section 3 Let $Z_{g}$ denote the 4-manifold obtained from $F \times G$ by performing -1 Luttinger surgeries on the $2 g$ disjoint Lagrangian tori $Y_{1} \times A_{i}$ and $Y_{2} \times B_{i}$ along the curves $\ell_{Y_{1} \times A_{i}}=\tilde{a}_{i}$ and $\ell_{Y_{2} \times B_{i}}=\tilde{b}_{i}$. Then by Theorem 1 the fundamental group of $Z_{g}$ is generated by loops $\tilde{x}_{1}, \tilde{y}_{1}, \tilde{x}_{2}, \tilde{y}_{2}, \tilde{a}_{1}, \tilde{b}_{1}, \ldots, \tilde{a}_{g}, \tilde{b}_{g}$ and the relations

$$
\left[\tilde{x}_{1}, \tilde{b}_{i}\right]=\tilde{a}_{i}, \quad\left[\tilde{x}_{2}, \tilde{a}_{i}\right]=\tilde{b}_{i}
$$

hold in $\pi_{1}\left(Z_{g}\right)$. Moreover, the standard symplectic generators for $\pi_{1}(F)$ are sent to $\tilde{x}_{1}, \tilde{y}_{1}, \tilde{x}_{2}, \tilde{y}_{2}$ in $\pi_{1}\left(Z_{g}\right)$.

Since the meridian $\mu_{F_{B}}$ of $F_{B} \subset B$ is trivial, the symplectic sum of $B$ with $Z_{g}$ along their genus 2 symplectic surfaces $F_{B} \subset B$ and $F=F \times\{k\} \subset F \times G$,

$$
B_{g}=B \#_{F_{B}, F} Z_{g}
$$

has fundamental group a quotient of $\left(\mathbb{Z} t_{1} \oplus \mathbb{Z} t_{2}\right) * \pi_{1}\left(Z_{g}\right)$. We choose this symplectic sum so that the generators $a_{1}, b_{1}, a_{2}, b_{2}$ for $\pi_{1}\left(F_{B}\right)$ are identified (in order) with the generators $\tilde{x}_{1}, \tilde{y}_{1}, \tilde{x}_{2}, \tilde{y}_{2}$.

The fifth assertion of Theorem 7 shows that $\tilde{x}_{1}, \tilde{y}_{1}$, and $\tilde{x}_{2}$ are trivial in $\pi_{1}\left(B_{g}\right)$. The relations coming from the Luttinger surgeries then show that $\tilde{a}_{i}=1=\tilde{b}_{i}$. Since $b_{2}=\tilde{y}_{2}$ is identified with $t_{2}, \pi_{1}\left(B_{g}\right)$ is generated by $t_{1}$ and $t_{2}$. A calculation using the Mayer-Vietoris sequence shows that $H_{1}\left(B_{g}\right)=\mathbb{Z}^{2}$, and so $\pi_{1}\left(B_{g}\right)=\mathbb{Z} t_{1} \oplus \mathbb{Z} t_{2}$. Hence $\left(B_{g}, T_{1}, T_{2}\right)$ is a telescoping triple, as desired.

The Euler characteristic of $B_{g}$ is calculated as

$$
e\left(B_{g}\right)=e(B)+e(F \times G)+4=6+4 g-4+4=6+4 g,
$$

and the signature is computed by Novikov additivity: $\sigma\left(B_{g}\right)=\sigma(B)=-2$.

The torus $H_{1}$ in $B$ geometrically dual to $F_{B}$ can be lined up with one of the parallel copies $\{z\} \times G$ in $F \times G$ (i.e. take a relative symplectic sum, [15]) to produce a square -1 genus $g+1$ surface in $B_{g}$.

Minimality follows from [6, Lemma 2], which shows that $Z_{g}$ is minimal (its universal cover is contractible, so $\pi_{2}\left(Z_{g}\right)=0$ ), and from Usher's theorem (Theorem 5 . 
We can also produce telescoping triples with odd signature starting with $B$. Recall that a symplectic 4-manifold $X$ containing a symplectic surface $F$ is called relatively minimal if every -1 sphere in $X$ intersects $F$.

Lemma 10. The blowup $A=B \# \overline{\mathbb{C P}}^{2}$ contains a genus 3 symplectic surface $F_{3}$ with trivial normal bundle and two Lagrangian tori $T_{1}$ and $T_{2}$ so that the surfaces $F_{3}, T_{1}, T_{2}$ are pairwise disjoint, $\left(A, F_{3}\right)$ is relatively minimal, and:

(1) $\pi_{1}\left(A-\left(F_{3} \cup T_{1} \cup T_{2}\right)\right)=\mathbb{Z}^{2}$, generated by $t_{1}$ and $t_{2}$.

(2) The inclusion $A-\left(F_{3} \cup T_{1} \cup T_{2}\right) \subset A$ induces an isomorphism on fundamental groups. In particular, the meridians $\mu_{F_{3}}, \mu_{T_{1}}, \mu_{T_{2}}$ all vanish in $\pi_{1}\left(A-\left(F_{3} \cup T_{1} \cup T_{2}\right)\right)$.

(3) The Lagrangian push-offs $m_{T_{1}}, \ell_{T_{1}}$ of $\pi_{1}\left(T_{1}\right)$ are sent to 1 and $t_{2}$ respectively in the fundamental group of $A-\left(F_{3} \cup T_{1} \cup T_{2}\right)$.

(4) The Lagrangian push-offs $m_{T_{2}}, \ell_{T_{2}}$ of $\pi_{1}\left(T_{2}\right)$ are sent to $t_{1}$ and $t_{2}$ respectively in the fundamental group of $A-\left(F_{3} \cup T_{1} \cup T_{2}\right)$.

(5) There is a standard symplectic generating set $\left\{a_{1}, b_{1}, a_{2}, b_{2}, a_{3}, b_{3}\right\}$ for $\pi_{1}\left(F_{3}\right)$ so that the push-off $F_{3} \subset A-\left(F_{3} \cup T_{1} \cup T_{1}\right)$ takes $b_{2}$ to $t_{2}, b_{3}$ to $t_{1}$, and all other generators to 1 .

In particular, $\left(A, T_{1}, T_{2}\right)$ is a telescoping triple.

Proof. The 4-manifold $B$ of Theorem 7 contains a symplectic genus 2 surface $F$ of square zero and a geometrically dual symplectic torus $H_{1}$ of square -1 . Symplectically resolve the union $F \cup H_{1}$ to get $F_{3}^{\prime}$, a genus 3 symplectic surface in $B$ which misses $T_{1}$ and $T_{2}$. The surface $F_{3}^{\prime}$ has square $\left(F+H_{2}\right)^{2}=1$. Blow up $B$ at one point on $F_{3}^{\prime}$ to construct $A$ and denote the proper transform of $F_{3}^{\prime}$ by $F_{3}$.

Since $F_{3}$ has a geometrically dual 2 -sphere (the exceptional sphere), the meridian of $F_{3}$ in $A-F_{3} \subset F_{3}$ is nullhomotopic. The rest of the fundamental group assertions follow from Theorem 7 .

Although $A$ is not minimal, T.-J. Li's theorem (Theorem 6) implies that every -1 sphere in $A$ intersects $F_{3}$, since $B$ is minimal, and neither rational nor ruled.

Note that Luttinger surgery on $T_{1}$ and $T_{2}$ in $A$ produces a symplectic 4-manifold homeomorphic to $\mathbb{C P}^{2} \# 4 \overline{\mathbb{C P}}^{2}$, but this manifold is not minimal; it is just the blowup $X_{1,3} \# \overline{\mathbb{C P}}^{2}$. We do not know how to produce a minimal symplectic 4-manifold with this homeomorphism type.

We next produce a 4-manifold $C$ with $e=8$ and $\sigma=-4$ by stopping the construction of a minimal symplectic 4-manifold homeomorphic to $\mathbb{C P}^{2} \# 5 \overline{\mathbb{C P}}^{2}$ in the proof of [6, Theorem 10] before the last two Luttinger surgeries to obtain the following.

Theorem 11. There exists a minimal telescoping triple $\left(C, T_{1}, T_{2}\right)$ with $e(C)=8$ and $\sigma(C)=-4$. Moreover, $C$ contains a square -1 torus disjoint from $T_{1} \cup T_{2}$.

Proof. We follow the notation and proof of [6. Theorem 10]. By not performing the Luttinger surgeries on the tori $T_{3}$ and $T_{4}$, one obtains a minimal symplectic 4-manifold $C$ such that $\pi_{1}\left(C-\left(T_{3} \cup T_{4}\right)\right)$ is generated by the two commuting elements $y$ and $a_{2}$. The MayerVietoris sequence shows that $H_{1}\left(C-\left(T_{3} \cup T_{4}\right) ; \mathbb{Z}\right)=\mathbb{Z}^{2}$, and so $\pi_{1}\left(C-\left(T_{1} \cup T_{2}\right)\right)=$ 
$\mathbb{Z} y \oplus \mathbb{Z} a_{2}$. The meridians and Lagrangian push-offs of $T_{3}$ and $T_{4}$ are given by $\mu_{T_{3}}=1$, $m_{T_{3}}=1, \ell_{T_{3}}=a_{2}$ and $\mu_{T_{4}}=1, m_{T_{4}}=y, \ell_{T_{4}}=a_{2}$. Thus $\left(C, T_{3}, T_{4}\right)$ is a telescoping triple. We relabel $T_{3}$ as $T_{1}$ and $T_{4}$ as $T_{2}$.

The -1 torus comes about from the construction. Briefly, $C$ is obtained by performing Luttinger surgeries on the symplectic sum $\left(T^{2} \times F_{2}\right) \#_{S}\left(\left(T^{2} \times S^{2}\right) \# 4 \overline{\mathbb{C P}}^{2}\right)$ along the genus 2 surface $\{x\} \times F_{2}$ in $T^{2} \times F_{2}$ and the genus 2 surface $F_{2}^{\prime} \subset\left(T^{2} \times S^{2}\right) \# 4 \overline{\mathbb{C P}}^{2}$ obtained by resolving the singularities of $\left(T^{2} \times\left\{p_{1}\right\}\right) \cup\left(\{q\} \times S^{2}\right) \cup\left(T^{2} \times\left\{p_{2}\right\}\right)$ and blowing up four times at points on this genus 2 surface. One can choose a square zero torus of the form $T^{2} \times\{y\} \subset T^{2} \times F_{2}$ which matches up (i.e. take a relative symplectic sum) with one of the four exceptional curves to provide a -1 symplectic torus disjoint from the Lagrangian tori where the Luttinger surgeries are performed.

The symplectic 4-manifold $X_{1,5}$ obtained from $C$ by +1 Luttinger surgeries on $T_{1}$ and $T_{2}$ as in Proposition 4 is minimal and homeomorphic to $\mathbb{C P}^{2} \# 5 \mathbb{C P}^{2}$.

Our next small model is a minimal telescoping triple built in the process of constructing a minimal symplectic 4-manifold homeomorphic to $\mathbb{C P}^{2} \# 7 \overline{\mathbb{C P}}^{2}$ in [6, Theorem 8]. One stops the construction before performing the two Luttinger surgeries, and these unused tori provide the desired $T_{1}$ and $T_{2}$.

Theorem 12. There exists a minimal telescoping triple $\left(D, T_{1}, T_{2}\right)$ with $e(D)=10$ and $\sigma(D)=-6$. Moreover, $D$ contains a square -1 torus disjoint from $T_{1} \cup T_{2}$.

Proof. The proof is similar to that of Theorem 11. We follow the notation and proof of [6, Theorem 8]. The 4-manifold $S$ contains two Lagrangian tori $T_{1}, T_{2}$ such that $\pi_{1}\left(S-\left(T_{1} \cup T_{2}\right)\right)$ is generated by the two commuting elements $s_{1}, t_{1}$. The Mayer-Vietoris sequence computes $H_{1}\left(S-\left(T_{1} \cup T_{2}\right)\right)=\mathbb{Z}^{2}$ so that $\pi_{1}\left(S-\left(T_{1} \cup T_{2}\right)\right)=\mathbb{Z} s_{1} \oplus \mathbb{Z} t_{1}$.

The meridians and Lagrangian push-offs of $T_{1}$ and $T_{2}$ are given by $\mu_{T_{1}}=1, m_{T_{1}}=s_{1}$, $\ell_{T_{1}}=s_{1}^{-1}$ and $\mu_{T_{2}}=1, m_{T_{2}}=t_{1}, \ell_{T_{2}}=s_{1}$. Thus $\left(S, T_{1}, T_{2}\right)$ is a telescoping triple. It is shown to be minimal in the proof of [6, Theorem 8]. The existence of a square -1 torus follows exactly as in the proof of Theorem 11 , since the manifold $S$ is obtained by Luttinger surgeries on the symplectic sum of $\left(T^{2} \times T^{2}\right) \# 2 \overline{\mathbb{C P}}^{2}$ and $\left(T^{2} \times S^{2}\right) \# 4 \overline{\mathbb{C P}}^{2}$ along a genus 2 surface. Relabel $S$ as $D$.

The symplectic 4-manifold $X_{1,7}$ obtained from $D$ by +1 Luttinger surgeries on $T_{1}$ and $T_{2}$ as in Proposition 4 is minimal and homeomorphic to $\mathbb{C P}^{2} \# 7 \overline{\mathbb{C P}^{2}}$ ([6]). More generally, the following proposition is true.

Proposition 13. Let $X$ be one of the manifolds $B, B_{g}, C, D$ and $T_{1}, T_{2}$ the corresponding Lagrangian tori as described in Theorems 7, 11, 12, with Lagrangian push-offs $m_{T_{i}}$ and $\ell_{T_{i}}$ (and trivial meridians). Then the symplectic 4-manifolds obtained from \pm 1 Luttinger surgery on one or both of $T_{1}, T_{2}$ along $m_{T_{i}}$ or $\ell_{T_{i}}$ are all minimal.

We omit the proof, which is based on Usher's theorem and a repeated use of [6. Lemma 2]. The reader may look at the proofs of Theorems 8, 10, and 13 of [6].

Since our emphasis in this article is on 4-manifolds with odd intersection forms, we recall the following result [14, Theorem VII.3.2 for minimality]. 
Theorem 14. The symplectic manifold $E^{\prime}(k)=E(k)_{2,3}$ obtained from the elliptic surface $E(k)$ by performing two log transforms of order 2 and 3 is simply connected and minimal. It has Euler characteristic $e\left(E^{\prime}(k)\right)=12 k$, signature $\sigma\left(E^{\prime}(k)\right)=-8 k$, and an odd intersection form.

\section{Minimal symplectic 4-manifolds with $\sigma=-3$ and $e \geq 15$}

The most complicated examples we construct are simply connected minimal symplectic 4-manifolds with signature -3 . Putting these in the context of telescoping triples is more trouble than constructing them directly. Moreover, with the exception of the $\sigma=-3$ manifolds, our inductive scheme for filling out the entire geography for $\sigma \leq-2$ only requires at most one copy of the manifold $A$ of Lemma 10 . Hence in this section we prove the following theorem.

Theorem 15. For each integer $k \geq 2$, there exists a simply connected minimal symplectic 4-manifold $X_{1+2 k, 4+2 k}$ with $e\left(X_{1+2 k, 4+2 k}\right)=7+4 k$ and $\sigma\left(X_{1+2 k, 4+2 k}\right)=-3$.

The construction of 4-manifolds with signature -3 for $e=7+8 g$ is easier than for $e=11+8 g$. Roughly speaking, to produce a 4-manifold with $e=7+8 g$, we take the symplectic sum along a genus 3 surface of the 4-manifold $A$ of Lemma 10 with $F \times G$, where $F$ is a genus 3 surface and $G$ is a genus $g$ surface, and perform Luttinger surgeries on the Lagrangian tori in $G$. To produce a 4-manifold with $e=11+8 g$ requires producing a substitute $A^{\prime}$ for $A$ which has signature -3 and $e=11$, and which satisfies the conclusions of Lemma 10 To do this, we take the symplectic sum of $A$ with the product $F \times G$ of two genus 2 surfaces along a symplectic torus.

Lemma 16. There exists a minimal symplectic 4-manifold $Z$ with $e(Z)=4$ and $\sigma(Z)=0$ which contains eight homologically essential Lagrangian tori $S_{1}, \ldots, S_{8}$ (in fact each $S_{i}$ has a geometrically dual torus $S_{i}^{d}$ so that all other intersections are zero) so that $\pi_{1}\left(Z-\bigcup_{i} S_{i}\right)$ is generated by $x_{1}, y_{1}, x_{2}, y_{2}$ and $a_{1}, b_{1}, a_{2}, b_{2}$, and so that the meridians and Lagrangian push-offs are given by

- $S_{1}: \mu_{1}=\left[b_{1}^{-1}, y_{1}^{-1}\right], m_{1}=x_{1}, \ell_{1}=a_{1}$,

- $S_{2}: \mu_{2}=\left[x_{1}^{-1}, b_{1}\right], m_{2}=y_{1}, \ell_{2}=b_{1} a_{1} b_{1}^{-1}$,

- $S_{3}: \mu_{3}=\left[b_{2}^{-1}, y_{1}^{-1}\right], m_{3}=x_{1}, \ell_{3}=a_{2}$,

- $S_{4}: \mu_{4}=\left[x_{1}^{-1}, b_{2}\right], m_{4}=y_{1}, \ell_{4}=b_{2} a_{2} b_{2}^{-1}$,

- $S_{5}: \mu_{5}=\left[b_{1} a_{1}^{-1} b_{1}^{-1}, y_{2}^{-1}\right], m_{5}=x_{2}, \ell_{5}=b_{1}^{-1}$,

- $S_{6}: \mu_{6}=\left[x_{2}^{-1}, b_{1} a_{1} b_{1}^{-1}\right], m_{6}=y_{2}, \ell_{6}=b_{1} a_{1} b_{1}^{-1} a_{1}^{-1} b_{1}^{-1}$,

- $S_{7}: \mu_{7}=\left[b_{2} a_{2}^{-1} b_{2}^{-1}, y_{2}^{-1}\right], m_{7}=x_{2}, \ell_{7}=b_{2}^{-1}$,

- $S_{8}: \mu_{8}=\left[x_{2}^{-1}, b_{2} a_{2} b_{2}^{-1}\right], m_{8}=y_{2}, \ell_{8}=b_{2} a_{2} b_{2}^{-1} a_{2}^{-1} b_{2}^{-1}$.

Proof. Proposition 7 of [6] (see also the construction of the manifold $P$ in [5]) computes the fundamental group of the complement of four Lagrangian tori $S_{1}, S_{2}, S_{3}, S_{4}$ in the product $F_{1} \times G$ of a punctured torus $F_{1}$ with a genus 2 surface $G$. This group is generated 
by loops $x_{1}, y_{1}, a_{1}, b_{1}, a_{2}, b_{2}$ (called $\tilde{x}, \tilde{y}, \tilde{a}_{1}, \tilde{b}_{1}, \tilde{a}_{2}, \tilde{b}_{2}$ there) where $a_{1}, b_{1}, a_{2}, b_{2}$ are a standard generating set for $\pi_{1}(G)$, and $x_{1}, y_{1}$ are a standard generating set for $\pi_{1}\left(F_{1}\right)$ based at a point $h$ on the boundary. In particular, the copy $\{h\} \times G$ in the boundary of $F_{1} \times G$ is carries the loops $a_{1}, b_{1}, a_{2}, b_{2}$.

We take two copies of this manifold, calling the second $F_{2} \times G$, its tori $S_{5}, S_{6}, S_{7}, S_{8}$, and its generators $x_{2}, y_{2}, a_{1}^{\prime}, b_{1}^{\prime}, a_{2}^{\prime}, b_{2}^{\prime}$. Glue the two copies together using a diffeomorphism of their boundary of the form Id $\times \phi: \partial F_{2} \times G \rightarrow \partial F_{1} \times G$, where $\phi: G \rightarrow G$ is the basepoint preserving diffeomorphism inducing the map

$$
\left(a_{1}^{\prime}, b_{1}^{\prime}, a_{2}^{\prime}, b_{2}^{\prime}\right) \mapsto\left(b_{1}^{-1}, b_{1} a_{1} b_{1}^{-1}, b_{2}^{-1}, b_{2} a_{2} b_{2}^{-1}\right)
$$

(a composite of six Dehn twists: see [6, Lemma 9]).

The resulting manifold $Z$ can also be described as the symplectic sum of two copies of a product of a genus 1 and a genus 2 surface. Thus the result is symplectic and the 8 tori are Lagrangian. The tori $S_{1}, S_{2}, S_{3}, S_{4}$ in $F_{1} \times G$ have geometrically dual tori $S_{1}^{d}, S_{2}^{d}, S_{3}^{d}, S_{4}^{d}$ which form a direct sum (geometrically) of four hyperbolic pairs, and similarly for $S_{5}, S_{6}, S_{7}, S_{8}$. Clearly $e(Z)=4$ and $\sigma(Z)=0$. Applying the SeifertVan Kampen theorem to the formulae of Proposition 7 of [6] yields the fundamental group assertions. Since the diffeomorphism Id $\times \phi: \partial F_{2} \times G \rightarrow \partial F_{1} \times G$ extends to $F_{2} \times G \rightarrow F_{1} \times G$, the manifold $Z$ is just the product of two genus 2 surfaces. In particular $Z$ is minimal.

Let $Y$ be the symplectic 4-manifold obtained from the manifold $Z$ of Lemma 16 by performing the following seven Luttinger surgeries on $S_{1}, \ldots, S_{7}$ :

(1) $S_{1}:+1$ surgery along $m_{1}$.

(2) $S_{2}:+1$ surgery along $\ell_{2}$.

(3) $S_{3}:+1$ surgery along $\ell_{3}$.

(4) $S_{4}:+1$ surgery along $m_{4}$

(5) $S_{5}:+1$ surgery along $\ell_{5}$.

(6) $S_{6}:+1$ surgery along $m_{6}$.

(7) $S_{7}:+1$ surgery along $m_{7}$

Since the torus $S_{8}$ has not been surgered, it remains as a Lagrangian torus in $Y$. Since $S_{8}$ is homologically essential, the symplectic form can be perturbed so that $S_{8}$ becomes symplectic. The symplectic 4-manifold $Y$ is minimal, since it is a symplectic sum of manifolds with contractible universal cover (see [6, Lemma 2]).

Let $\left(B, T_{1}, T_{2}\right)$ be the telescoping triple of Theorem 7 , with $B$ containing the genus 2 symplectic surface $F$ and geometrically dual -1 torus $H_{1}$. Perform +1 Luttinger surgery on $T_{2}$ along $m_{T_{2}}$ to kill $t_{1}$, yielding a minimal (Proposition 13 symplectic 4-manifold $\hat{B}$. Note that $\hat{B}$ still contains the three surfaces $T_{1}, F, H_{1}$ and $\pi_{1}\left(\hat{B}-\left(T_{1} \cup F \cup H_{1}\right)\right)=\mathbb{Z} t_{2}$. The torus $T_{1}$ is disjoint from the geometrically dual symplectic surfaces $F$ and $H_{1}$, and its Lagrangian push-offs are $m_{T_{1}}=1$ and $\ell_{T_{1}}=t_{2}$ by Theorem 7 .

Lemma 17. The symplectic sum $X_{3,5}=\hat{B} \#_{T_{1}, S_{8}} Y$ is simply connected, minimal, and contains a symplectic genus 2 surface of square 0 and a geometrically dual symplectic torus of square -1 . Moreover, $e\left(X_{3,5}\right)=10$ and $\sigma\left(X_{3,5}\right)=-2$, so that $X_{3,5}$ is homeomorphic but not diffeomorphic to $3 \mathbb{C P}^{2} \# 5 \overline{\mathbb{C P}}^{2}$. 
Proof. We refer to the notation in the statement of Theorem 7 . The fundamental group of $X_{3,5}-F$ is generated by $t_{2}, x_{1}, y_{1}, x_{2}, y_{2}, a_{1}, b_{1}, a_{2}, b_{2}$ by the Seifert-Van Kampen theorem (recall that $t_{1}$ is killed by Luttinger surgery on $T_{2}$ ).

Since the meridian of $T_{1}$ in $\pi_{1}\left(B-\left(F \cup T_{1} \cup T_{2}\right)\right)$ is trivial, $\mu_{8}$ is trivial in $\pi_{1}\left(X_{3,5}-F\right)$. Choose the gluing map $S_{8} \rightarrow T_{1}$ so that $\ell_{8}$ is killed and $m_{8}$ is sent to $t_{2}$ (i.e. $m_{8} \mapsto$ $\left.\ell_{T_{1}}, \ell_{8} \mapsto m_{T_{1}}^{-1}\right)$.

Since $\ell_{8}$ is a conjugate of $b_{2}^{-1}$ and $m_{8}=y_{2}$, it follows that $b_{2}=1$ and $y_{2}=t_{2}$. This implies that $\mu_{3}$ and $\mu_{4}$ are trivial, and hence the third and fourth Luttinger surgeries listed above show that $a_{2}=1$ and $y_{1}=1$. Thus $\mu_{1}$ and $\mu_{7}$ are killed. The first and seventh Luttinger surgeries now show that $x_{1}=1$ and $x_{2}=1$. Continuing, we see that $\mu_{2}$ and $\mu_{6}$ are killed so that the corresponding surgeries give $a_{1}=1$ and $y_{2}=1$. This implies $\mu_{5}=1$ and so $b_{1}=1$. Hence $\pi_{1}\left(X_{3,5}\right)=1$.

The minimality of $X_{3,5}$ follows from Usher's theorem. The genus 2 surface $F$ and torus $H_{1}$ in $B$ survive to give the required surfaces in $X_{3,5}$.

Proof of Theorem 15 . We define two minimal simply connected symplectic 4-manifolds: let $X_{-}=X_{3,5}$ and let $X_{+}=X_{1,3}$ (thus $X_{+}$is obtained from the manifold $\hat{B}$ defined above by performing +1 Luttinger surgery on $T_{1}$ along $\ell_{T_{1}}$; see Corollary 8 ). Then $X_{-}$ and $X_{+}$each contain a symplectic genus 3 surface $F_{3}$ of square 1 obtained by resolving the union $H_{1} \cup F$. Moreover, $e\left(X_{-}\right)=10, \sigma\left(X_{-}\right)=-2, e\left(X_{+}\right)=6$, and $\sigma\left(X_{+}\right)=-2$.

Blow up $X_{ \pm}$once at a point on $F_{3}$ and take the proper transform. Call the result $\tilde{X}_{ \pm}$ and denote by $\tilde{F}_{3}$ the proper transform of $F_{3}$. Thus $\tilde{F}_{3}$ is a genus 3 , square zero symplectic surface with simply connected complement, which meets every -1 sphere in $\tilde{X}_{ \pm}$since $X_{ \pm}$is minimal.

We now mimic the proof of Corollary 9 Take the product $F_{3} \times G$ of a genus 3 surface with a genus $g$ surface. Perform Luttinger surgeries on the $2 g$ disjoint Lagrangian tori $Y_{1} \times A_{j}$ and $Y_{2} \times B_{j}$ along the curves $\ell_{Y_{1} \times A_{j}}=a_{j}$ and $\ell_{Y_{2} \times B_{j}}=b_{j}$ to obtain a manifold $Z_{g}$.

Then by Theorem 1 the fundamental group of $Z_{g}$ is generated by the $6+2 g$ loops $\tilde{x}_{1}, \tilde{y}_{1}, \tilde{x}_{2}, \tilde{y}_{2}, \tilde{x}_{3}, \tilde{y}_{3}, \tilde{a}_{1}, \tilde{b}_{1}, \ldots, \tilde{a}_{g}, \tilde{b}_{g}$, and the relations

$$
\left[\tilde{x}_{1}, \tilde{b}_{j}\right]=\tilde{a}_{j}, \quad\left[\tilde{x}_{2}, \tilde{a}_{j}\right]=\tilde{b}_{j}, \quad j=1, \ldots, g
$$

hold in $\pi_{1}\left(Z_{g}\right)$. Moreover, the standard symplectic generators for $\pi_{1}(F)$ are sent to $\tilde{x}_{1}$, $\tilde{y}_{1}, \tilde{x}_{2}, \tilde{y}_{2}, \tilde{x}_{3}, \tilde{y}_{3}$ in $\pi_{1}\left(Z_{g}\right)$.

Since $\pi_{1}\left(\tilde{X}_{ \pm}-\tilde{F}_{3}\right)=1$, the fundamental group of the symplectic sum

$$
Q_{ \pm, g}=\tilde{X}_{ \pm} \#_{\tilde{F}_{3}, F} Z_{g}
$$

is trivial. Indeed, the $\tilde{x}_{i}, \tilde{y}_{i}$ are killed by taking the symplectic sum, and the relations coming from the Luttinger surgeries show the $\tilde{a}_{j}$ and $\tilde{b}_{j}$ are killed also.

Now $Q_{ \pm, g}$ is minimal provided $g \geq 1$ by Usher's theorem since $\tilde{X}_{ \pm}$is relatively minimal by Li's theorem (Theorem 6 . 
One computes:

$$
\begin{aligned}
& e\left(Q_{-, g}\right)=e\left(\tilde{X}_{-}\right)+e\left(Z_{g}\right)+8=11+8 g-8+8=11+8 g, \\
& \sigma\left(Q_{-, g}\right)=\sigma\left(\tilde{X}_{-}\right)+\sigma\left(Z_{g}\right)=-3,
\end{aligned}
$$

and

$$
\begin{aligned}
& e\left(Q_{+, g}\right)=e\left(\tilde{X}_{+}\right)+e\left(Z_{g}\right)+8=7+8 g-8+8=7+8 g, \\
& \sigma\left(Q_{+, g}\right)=\sigma\left(\tilde{X}_{+}\right)+\sigma\left(Z_{g}\right)=-3 .
\end{aligned}
$$

Thus we set $X_{1+2 k, 4+2 k}=Q_{+, k / 2}$ if $k$ is even and $X_{1+2 k, 4+2 k}=Q_{-,(k-1) / 2}$ if $k$ is odd. This completes the proof of Theorem 15 .

Remark 1. In the construction of the manifold $X_{5,8}$, the first step (see the paragraph preceding Lemma 17) involves Luttinger surgery on the torus $T_{2}$ to kill $t_{1}$. If one constructs the manifold $P_{5,8}$ following the same construction as for $X_{5,8}$ without performing this surgery, then $P_{5,8}$ is a minimal symplectic 4-manifold with $\pi_{1}\left(P_{5,8}\right)=\mathbb{Z} t_{1}$ containing an essential Lagrangian (or if desired symplectic) torus $T=T_{2}$ such that the inclusion map $\pi_{1}(T) \rightarrow \pi_{1}\left(P_{5,8}\right)$ is a surjection and the inclusion map $\pi_{1}\left(P_{5,8}-T\right) \rightarrow \pi_{1}\left(P_{5,8}\right)$ an isomorphism. Moreover, $e\left(P_{5,8}\right)=15$ and $\sigma\left(P_{5,8}\right)=-3$.

More generally, for any $k \geq 2$ the same construction yields a minimal symplectic 4manfold $P_{1+2 k, 4+2 k}$ containing a Lagrangian or symplectic torus $T$ with these properties and such that $e\left(P_{1+2 k, 4+2 k}\right)=7+4 k, \sigma\left(P_{1+2 k, 4+2 k}\right)=-3$.

\section{Small examples with odd signature}

In this section, we remind the reader of some known examples of small manifolds with odd signature, and construct a few new ones.

Kotschick showed in [19] that the Barlow surface is smoothly irreducible and hence it is a minimal symplectic 4-manifold homeomorphic to $\mathbb{C P}^{2} \# 8 \overline{\mathbb{C P}^{2}}$. This manifold realizes the pair $e=11, \sigma=-7$.

In [15], Gompf constructs small minimal symplectic 4-manifolds which contain appropriate tori. For example, the manifold Gompf calls $S_{1,1}$ is minimal, has $e=23$ and $\sigma=-15$, and contains a symplectic torus of square zero with simply connected complement ([15] Lemma 5.5]). The minimality of $S_{1,1}$ was proved by Stipsicz [31].

Gompf also constructs other minimal symplectic 4-manifolds: the manifold $R_{2,1}$ has $e=21$ and $\sigma=-13$ and $R_{2,2}$ has $e=19$ and $\sigma=-11$. The minimality of $R_{2,1}$ was proved by J. Park [27], and $R_{2,2}$ was proved to be minimal by Szabó [34].

In [33], Stipsicz and Szabó construct a minimal symplectic 4-manifold homeomorphic to $\mathbb{C P}^{2} \# 6 \overline{\mathbb{C P}}^{2}$, realizing $e=9, \sigma=-5$.

In [25], the fifth author constructs a minimal simply connected symplectic 4-manifold homeomorphic to $3 \mathbb{C P}^{2} \# 12 \overline{\mathbb{C P}}^{2}$, hence with $e=17$ and $\sigma=-9$, containing a symplectic torus $T_{2,4}$ with simply connected complement. This manifold is called $X_{12}$ in that article; we will use the notation $X_{3,12}$ here to avoid confusion.

We produce a few more small examples. 
Proposition 18. There exists a minimal simply connected symplectic 4-manifold $X_{5,10}$ homeomorphic to $5 \mathbb{C P}^{2} \# 10 \overline{\mathbb{C P}}^{2}$, hence with $e=17$ and $\sigma=-5$.

Proof. The manifold $X_{1,3}$ of Corollary 8 contains a symplectic genus 2 surface $F$ of square zero, and a geometrically dual symplectic torus $H_{1}$ with square -1 . Symplectically resolve $F \cup H_{1}$ to produce a square 1 symplectic genus 3 surface $F_{3} \subset X_{1,3}$.

Blow up $X_{1,3}$ at a point on $F_{3}$ to obtain $\tilde{X}_{1,3}$ and take $\tilde{F}_{3}$ to be the proper transform of $F_{3}$. Then $\tilde{F}_{3}$ is a square zero symplectic surface that meets every -1 sphere in $\tilde{X}_{1,3}$ by Li's theorem (Theorem 6). Moreover, since $X_{1,3}$ is simply connected and $\tilde{F}_{3}$ meets the exceptional sphere, $\tilde{X}_{1,3}-\tilde{F}_{3}$ is simply connected.

Take $Y=T \times F_{2}$, the product of a torus with a genus 2 surface. Then $Y$ contains the geometrically dual symplectic surfaces $T \times\{p\}$ and $\{q\} \times F_{2}$. Symplectically resolve their union to obtain a genus 3 , square 2 symplectic surface $F_{3}^{\prime} \subset Y$. Note that the homomorphism induced by inclusion $\pi_{1}\left(F_{3}^{\prime}\right) \rightarrow \pi_{1}(Y)$ is surjective. Blow up $Y$ twice at points on $F_{3}^{\prime}$ to obtain $\tilde{Y}$ and the proper transform $\tilde{F}_{3}^{\prime}$, a square zero genus 3 symplectic surface. Then the symplectic sum

$$
X_{5,10}=\tilde{X}_{1,3} \#_{\tilde{F}_{3}, \tilde{F}_{3}^{\prime}} \tilde{Y}
$$

is simply connected. It is minimal by Usher's theorem.

Its characteristic numbers are

$$
\begin{aligned}
e\left(X_{5,10}\right) & =e\left(\tilde{X}_{1,3}\right)+e(\tilde{Y})+8=7+2+8=17, \\
\sigma\left(X_{5,10}\right) & =\sigma\left(\tilde{X}_{1,3}\right)+\sigma(\tilde{Y})=-3-2=-5 .
\end{aligned}
$$

The proposition follows.

Proposition 19. There exists a minimal simply connected symplectic 4-manifold $X_{5,12}$ homeomorphic to $5 \mathbb{C P}^{2} \# 12 \overline{\mathbb{C P}}^{2}$, hence with $e=19$ and $\sigma=-7$.

Proof. The proof is very similar to the proof of Proposition 18 Construct $\tilde{X}_{1,3}$ and $\tilde{F}_{3}$ as in that proof.

Take $Z=T \times T$, the product of two tori. Pick three distinct points $p_{1}, p_{2}, q$ in $T$. Then $Z$ contains the three symplectic surfaces $T \times\left\{p_{1}\right\}, T \times\left\{p_{2}\right\}$ and $\{q\} \times T$. Symplectically resolve their union to obtain a genus 3 , square 4 symplectic surface $F_{3}^{\prime} \subset Z$. Note that the homomorphism induced by inclusion $\pi_{1}\left(F_{3}^{\prime}\right) \rightarrow \pi_{1}(Z)$ is surjective. Blow up $Z$ four times at points on $F_{3}^{\prime}$ to obtain $\tilde{Z}$ and the proper transform $\tilde{F}_{3}^{\prime}$, a square zero genus 3 symplectic surface.

Then the symplectic sum

$$
X_{5,12}=\tilde{X}_{1,3} \#_{\tilde{F}_{3}, \tilde{F}_{3}^{\prime}} \tilde{Z}
$$

is simply connected. It is minimal by Usher's theorem.

Its characteristic numbers are

$$
\begin{aligned}
& e\left(X_{5,12}\right)=e\left(\tilde{X}_{1,3}\right)+e(\tilde{Z})+8=7+4+8=19, \\
& \sigma\left(X_{5,12}\right)=\sigma\left(\tilde{X}_{1,3}\right)+\sigma(\tilde{Z})=-3-4=-7 .
\end{aligned}
$$

The proposition follows. 
Proposition 20. There exists a minimal simply connected symplectic 4-manifold $X_{5,14}$ homeomorphic to $5 \mathbb{C P}^{2} \# 14 \overline{\mathbb{C P}}^{2}$, hence with $e=21$ and $\sigma=-9$.

Proof. The proof is very similar to the proof of Proposition 19 Construct $\tilde{X}_{1,3}$ and $\tilde{F}_{3}$ as in that proof.

Take $Z=T \times S^{2}$, the product of a torus and a sphere. Pick three distinct points $p_{1}, p_{2}, p_{3}$ in $S^{2}$ and $q \in T$. Then $Z$ contains the four symplectic surfaces $T \times\left\{p_{1}\right\}$, $T \times\left\{p_{2}\right\}, T \times\left\{p_{3}\right\}$ and $\{q\} \times S^{2}$. Symplectically resolve their union to obtain a genus 3 , square 6 symplectic surface $F_{3}^{\prime} \subset Z$. Note that the homomorphism induced by inclusion $\pi_{1}\left(F_{3}^{\prime}\right) \rightarrow \pi_{1}(Z)$ is surjective. Blow up $Z$ six times at points on $F_{3}^{\prime}$ to obtain $\tilde{Z}$ and the proper transform $\tilde{F}_{3}^{\prime}$, a square zero genus 3 symplectic surface.

Then the symplectic sum

$$
X_{5,14}=\tilde{X}_{1,3} \#_{\tilde{F}_{3}, \tilde{F}_{3}^{\prime}} \tilde{Z}
$$

is simply connected. It is minimal by Usher's theorem.

Its characteristic classes are

$$
\begin{aligned}
& e\left(X_{5,14}\right)=e\left(\tilde{X}_{1,3}\right)+e(\tilde{Z})+8=7+6+8=21, \\
& \sigma\left(X_{5,14}\right)=\sigma\left(\tilde{X}_{1,3}\right)+\sigma(\tilde{Z})=-3-6=-9 .
\end{aligned}
$$

The proposition follows.

\section{The main theorem}

In this section we prove Theorem A stated in the introduction. We begin with an arithmetic lemma. The purpose of this lemma is to calculate the number of each of the model manifolds $B, B_{g}, C, D, E(k)$ needed to construct a 4-manifold with specified signature and Euler characterstic. The proof includes an algorithm for finding these numbers.

Lemma 21. Given any pair of non-negative integers $(m, n)$ such that

$$
0 \leq m \leq 4 n-1
$$

there exist non-negative integers $b, c, d, g$, and $k$ such that

$$
m=d+2 c+3 b+4 g \text { and } n=b+c+d+g+k
$$

and such that $b \geq 1$ if $g>0$.

Proof. If $m=0$, set $k=n$ and $b=c=d=g=0$.

Assume then that $m>0$. Choose a non-negative integer $\ell$ so that $(m+1) / 4 \leq n-\ell$ $\leq m$. Let

$$
s=\max \{z \in \mathbb{Z} \mid 3 z \leq 4 n-4 \ell-m-1\} \quad \text { and } \quad \Delta=4 n-4 \ell-m-1-3 s .
$$

Then $\Delta=0,1$ or 2 , and $s \geq 0$. Moreover,

$$
n-\ell-s-1=\frac{1}{3}(m-(n-\ell))-\frac{2}{3}+\frac{\Delta}{3} \geq \frac{\Delta}{3}-\frac{2}{3} \text {. }
$$


If $\Delta=0$, then set $b=1, c=0, d=s, g=n-\ell-s-1$, and $k=\ell$. Since $\Delta=0$, and since $g$ is an integer, $g \geq 0$.

If $\Delta=1$ and $s \geq 1$, then set $b=1, c=0, d=s-1, g=n-\ell-s-1$, and $k=\ell+1$. Note that $g \geq-1 / 3$ so that $g \geq 0$.

If $\Delta=1$ and $s=0$, then either $n-\ell-2 \geq 0$ in which case we set $b=2, c=$ $0, d=0, k=\ell$, and $g=n-\ell-2$, or else $n-\ell-2=-1$ in which case we take $b=0, c=1, d=0, k=\ell$, and $g=0$.

If $\Delta=2$ and $s \geq 2$, set $b=1, c=0, d=s-2, g=n-\ell-s-1$, and $k=\ell+2$.

If $\Delta=2, s=1$, and $n-\ell \geq 3$ then set $b=2, c=0, d=0, g=n-\ell-3$, and $k=\ell+1$. If $\Delta=2, s=1$, and $n-\ell<3$, then necessarily $n-\ell=2$, and so $(m, n)=(2, \ell+2)$ and we set $b=0, c=1, d=0, k=\ell+1$, and $g=0$.

This leaves the cases when $\Delta=2$ and $s=0$. If $n-\ell \geq 2$, set $b=1, c=1, d=$ $0, g=n-\ell-2$, and $k=\ell$. Finally, if $n-\ell=1$, then $(m, n)=(1,1+\ell)$, so we take $b=0, c=0, d=1, g=0$ and $k=\ell$.

We can now prove our main result. We state it in terms of $c_{1}^{2}=2 e+3 \sigma$ and $\chi_{h}=$ $\frac{1}{4}(e+\sigma)$ because it is simpler to work with these numbers than with pairs $(e, \sigma)$ where $e+\sigma \equiv 0(\bmod 4)$. Note that in this notation, a 4-manifold with $c_{1}^{2}=8 \chi_{h}+k$ has signature $k$, so the line $c_{1}^{2}=8 \chi_{h}-2$ corresponds to manifolds with signature -2 .

Theorem 22. For any pair $(c, \chi)$ of non-negative integers satisfying

$$
0 \leq c \leq 8 \chi-2
$$

with the possible exceptions of $(c, \chi)=(5,1),(9,2),(11,2)$, or $(13,2)$, there exists a minimal simply connected symplectic 4-manifold $Y=X_{2 \chi-1,10 \chi-c-1}$ with odd intersection form and

$$
c=c_{1}^{2}(Y) \text { and } \chi=\chi_{h}(Y) .
$$

Hence $Y$ is homeomorphic but not diffeomorphic to $(2 \chi-1) \mathbb{C P}^{2} \#(10 \chi-c-1) \overline{\mathbb{C P}}^{2}$.

Proof. We make extensive use of the manifolds $A, B, B_{g}, C, D, E^{\prime}(k)$ of (respectively) Lemma 10, Theorem 7, Corollary 9, Theorem 11, Theorem 12, and Theorem 14 We will also use the sporadic examples of Section 7 .

We first realize all pairs with $c$ even. Let $(m, n)=\left(\frac{1}{2} c, \chi\right)$. Lemma 21 produces integers $b, c, d, g$, and $k$ such that $m=d+2 c+3 b+4 g$ and $n=b+c+d+g+k$ and with $b \geq 1$ if $g>0$.

Construct the symplectic sum along tori of

(1) $b$ copies of $B$ if $g=0$, or one copy of $B_{g}$ and $b-1$ copies of $B$ if $g \geq 1$,

(2) $c$ copies of $C$,

(3) $d$ copies of $D$.

More precisely, each of the manifolds $B, C, D$ contains two essential Lagrangian tori. Construct the symplectic sum $Z$ of these manifolds by chaining them together, using Proposition 3 to ensure that at each stage one has a telescoping triple. 
Specifically, if $g=0$ take

$$
Z=\underbrace{B \#_{S} \ldots \#_{s} B}_{b} \#_{S} \underbrace{C \#_{S} \cdots \#_{S} C}_{c} \#_{S} \underbrace{D \#_{S} \cdots \#_{S} D}_{d}
$$

and if $g \geq 1$ take

$$
Z=B_{g} \#_{s} \underbrace{B \#_{s} \cdots \#_{s} B}_{b-1} \#_{S} \underbrace{C \#_{s} \cdots \#_{s} C}_{c} \#_{s} \underbrace{D \#_{s} \cdots \#_{s} D}_{d}
$$

where $\#_{s}$ denotes the symplectic sum along the appropriate tori (perturbing the symplectic forms so that they become symplectic) according to the recipe of Proposition 3 , so that the two unused Lagrangian tori (which we relabel $T_{1}$ and $T_{2}$ ) make $\left(Z, T_{1}, T_{2}\right)$ a telescoping triple.

If $k=0$, then perform +1 Luttinger surgery on $T_{1}$ and $T_{2}$ to obtain a simply connected (according to Proposition 4) symplectic 4-manifold $Y$.

If $k \geq 1$ and one of $b, c, d$ is positive, perform +1 Luttinger surgery on $T_{2}$ in $Z$ and take the symplectic sum of the result with the elliptic surface $E(k)$ along $T_{1}$ and a generic fiber $T$ of $E(k)$ to obtain the manifold $Y$. Since $E(k)-T$ is simply connected, so is $Y$, by the same reasoning as in the proof of Theorem 15 . Since $B, C$, and $D$ contain -1 tori disjoint from the Lagrangian tori $T_{1}, T_{2}$, the manifold $Y$ has odd intersection form.

If $k \geq 1$ and $b, c, d$ are zero, take $Y=E^{\prime}(k)$ (see Theorem 14, which has an odd intersection form.

Thus $Y$ is a simply connected symplectic manifold realizing the pair $(c, \chi)$. Since each of the manifolds $B, B_{g}, C$, and $D$ contains a surface of odd square which misses the tori used in forming the symplectic sums, and since $E^{\prime}(k)$ has an odd intersection form, it follows that $Y$ has an odd intersection form.

Since the 4-manifold $Y$ has indefinite, odd intersection form, Freedman's theorem [13] implies that $Y$ is homeomorphic to an appropriate connected sum of $\mathbb{C P}^{2}$ s and $\overline{\mathbb{~}}^{2}$ s.

Now we turn to the case when $c$ is odd. Suppose first that $1 \leq c \leq 8 \chi-17$. Let $\left(c^{\prime}, \chi^{\prime}\right)=(c-1, \chi-2)$. Thus $0 \leq c^{\prime} \leq 8 \chi^{\prime}-2$, and $c^{\prime}$ is even. Construct the manifold $Z$ corresponding to the pair $\left(c^{\prime}, \chi^{\prime}\right)$ and either perform +1 Luttinger surgery on $T_{1}$ or take the symplectic sum with $E(k)$ if $k \geq 1$. But rather than performing +1 Luttinger surgery on $T_{2}$ as we did above, perturb the symplectic form to make $T_{2}$ symplectic, and then take the symplectic sum with Gompf's manifold $S_{1,1}$ (see Section 7 p along the symplectic torus in $S_{1,1}$ with simply connected complement. Since $S_{1,1}$ has $c_{1}^{2}=1$ and $\chi_{h}=2$ the resulting symplectic manifold $Y$ has $\left(c_{1}^{2}, \chi_{h}\right)=(c, \chi)$.

Next suppose that $c$ is odd and $7 \leq c \leq 8 \chi-11$. Set $\left(c^{\prime}, \chi^{\prime}\right)=(c-7, \chi-2)$. Thus $0 \leq c^{\prime} \leq 8 \chi^{\prime}-2$ and $c^{\prime}$ is even. Construct the manifold $Z$ corresponding to the pair $\left(c^{\prime}, \chi^{\prime}\right)$. We repeat the argument of the previous paragraph, replacing Gompf's manifold $S_{1,1}$ with the manifold $X_{3,12}$ of Section 7 Take the symplectic sum of $Z$ with $X_{3,12}$ along $T_{2}$ and $T_{2,4}$. Since $c_{1}^{2}\left(X_{3,12}\right)=7$ and $\chi_{h}\left(X_{3,12}\right)=2$, the resulting manifold $Y$ realizes the pair $(c, \chi)$.

To realize all pairs $(c, \chi)$ with $c$ odd and $21 \leq c \leq 8 \chi-5$, repeat the argument once more, this time using the manifold $P_{5,8}$ described in Remark 1 at the end of the proof of 
Theorem 15. which has $c_{1}^{2}=21$ and $\chi_{h}=3$. A bit of care must be taken to ensure that the result is simply connected since $\pi_{1}\left(P_{5,8}\right)=\mathbb{Z}$. This is accomplished by making sure that the generator of $\pi_{1}(T)$ sent to the generator of $\pi_{1}\left(P_{5,8}-T\right)$ is identified with an element in the kernel of $\pi_{1}\left(T_{2}\right) \rightarrow \pi_{1}\left(Z-T_{2}\right)$ when forming the symplectic sum $Y=Z \#_{s} P_{5,8}$.

The manifold $Y=X_{1+2 k, 4+2 k}$ of Theorem 15 provides an example realizing the pair $(c, \chi)=(5+8 k, 1+k)$ for any $k \geq 2$, i.e. $21 \leq c=8 \chi-3$.

Since $c_{1}^{2} \equiv \sigma(\bmod 2)$, and simply connected 4-manifolds with odd signature have an odd intersection form, it follows that the manifolds constructed for $c$ odd also have an odd intersection form.

It remains to show that $Y$ is minimal. Since $E^{\prime}(k)$ is minimal, we assume that $c_{1}^{2}>0$. By Proposition 13, the 4-manifold obtained by performing one or two \pm 1 Luttinger surgeries on $T_{1}$ or $T_{2}$ along $\ell_{T_{i}}$ or $m_{T_{i}}$ in $B, C$, or $D$ is minimal. The $E(k)$ are minimal for $k \geq 2$. Although $E(1)$ is not minimal, every -1 sphere intersects the generic torus fiber. Thus $Y$ is the symplectic sum of minimal (or, if $k=1$, relatively minimal) symplectic 4-manifolds and therefore is minimal by Usher's theorem.

It is easy to check that the only pairs $(c, \chi)$ with $0 \leq c \leq 8 \chi-2$ which are omitted by these cases are

$$
\begin{aligned}
& (1,1),(3,1),(5,1) \\
& (1,2),(3,2),(5,2),(7,2),(9,2),(11,2),(13,2), \\
& (15,3),(17,3),(19,3)
\end{aligned}
$$

The examples listed in Section 7 realize most of these pairs. The only ones left unrealized are $(5,1),(9,2),(11,2)$, and $(13,2)$.

The four unrealized pairs do correspond to (non-minimal) symplectic 4-manifolds; e.g. blowups of $X_{1,3}$ or $X_{3,5}$. It is conjectured that one of the irreducible smooth 4-manifolds homeomorphic to $3 \mathbb{C P}^{2} \# 10 \overline{\mathbb{C P}}^{2}$ constructed in [25] and one of the irreducible smooth 4manifolds homeomorphic to $3 \mathbb{C P}^{2} \# 8 \overline{\mathbb{C P}}^{2}$ constructed in [29] are symplectic (and hence minimal): their Seiberg-Witten invariants have the right form to be the invariants of a minimal symplectic manifold.

There exist small simply connected minimal symplectic 4-manifolds with non-negative signatures (e.g. $\mathbb{C P}^{2}, S^{2} \times S^{2}$ ). To date, no small examples are known that contain a suitable Lagrangian torus for which we can extend the construction of Theorem 22. Some moderately large examples are known and we will briefly explore the consequences for the geography problem in the next section.

Remark 2. Each of the manifolds constructed in Theorem 22, with the possible exception of those corresponding $c_{1}^{2}=0$ and some of the small manifolds with $c$ odd, contains a nullhomologous torus suitable for altering the differentiable structure as explained in [8], using [24] to compute the change in Seiberg-Witten invariants. Those with $c_{1}^{2}=0$ are $E^{\prime}(k)$, for which the methods of [9, 10, 16] show how to alter the differentiable structure. Hence the manifolds of Theorem 22 admit infinitely many smooth structures. 
The proofs of Lemma 21 and Theorem 22 provide an algorithm for constructing simply connected minimal 4-manifolds with desired characteristic numbers, using the model manifolds $A, B, B_{g}, C, D$, and $E(k)$.

For example, to construct a minimal symplectic manifold homeomorphic but not diffeomorphic to $3 \mathbb{C P}^{2} \# 17 \overline{\mathbb{C P}}^{2}$, one sees that such a manifold would have $\left(c_{1}^{2}, \chi_{h}\right)=(2,2)$. This corresponds to $(m, n)=(1,2)$ in Lemma 21. In the notation of the proof of Lemma 21. we see that in this case $\ell=1, s=0$ and $\Delta=2$, so that $b=0, c=0, d=1, g=0$, and $k=1$. Thus the desired manifold is obtained by taking the symplectic sum

$$
D \#_{s} E^{\prime}(1)
$$

and performing +1 Luttinger surgery on the remaining Lagrangian torus in $D$.

As another example, we construct a minimal symplectic manifold homeomorphic but not diffeomorphic to $21 \mathbb{C P}^{2} \# 31 \overline{\mathbb{C P}^{2}}$, i.e. $\chi_{h}=11$ and $c_{1}^{2}=78$. Thus $(m, n)=(39,11)$. The proof of Lemma 21 provides $\ell=0, s=1$, and $\Delta=1$, and so $b=1, c=0, d=$ $0, g=9$, and $k=1$. Thus the desired manifold is obtained by taking the symplectic sum

$$
B_{9} \#_{S} E(1)
$$

and performing +1 Luttinger surgery on the remaining Lagrangian torus.

The integers produced by the algorithm in the proof of Lemma 21 are not unique. For example, the choice $b=2, c=0, d=1, g=8$, and $k=0$ yields a manifold

$$
B_{8} \#_{S} B \#_{S} D \text {. }
$$

Performing two +1 Luttinger surgeries on this manifold produces a (possibly different) minimal symplectic manifold homeomorphic to but not diffeomorphic to $21 \mathbb{C P}^{2} \# 31 \overline{\mathbb{C P}^{2}}$.

\section{Signature greater than -2}

Finding small minimal symplectic 4-manifolds with signature greater than -2 poses a special challenge. Stipsicz [32] shows how to produce simply connected minimal symplectic 4-manifolds with positive signature. The following theorem provides a method for producing many examples, given one. It is also useful in studying the geography problem for non-simply connected 4-manifolds.

To avoid an overly technical statement and proof, we separate the cases of $c$ odd and even, but a more complete statement would have similar hypotheses on $(c, \chi)$ as in Theorem 22 .

Theorem 23. Let $X$ be a symplectic 4-manifold and suppose that $X$ contains a symplectic torus $T$ such that the homomorphism $\pi_{1}(T) \rightarrow \pi_{1}(X)$ induced by inclusion is trivial. Then, for any pair $(c, \chi)$ of non-negative integers satisfying

$$
\begin{array}{ll}
0 \leq c \leq 8 \chi-2 & \text { if } c \text { is even, } \\
1 \leq c \leq 8 \chi-17 & \text { if } c \text { is odd }
\end{array}
$$


there exists a symplectic 4-manifold $Y$ with $\pi_{1}(Y)=\pi_{1}(X)$,

$$
c_{1}^{2}(Y)=c_{1}^{2}(X)+c \quad \text { and } \quad \chi_{h}(Y)=\chi_{h}(X)+\chi
$$

Moreover, if $X$ is minimal (or more generally if $(X, T)$ is relatively minimal) then the manifold $Y$ is minimal and has an odd, indefinite intersection form.

Proof. The argument is similar to the proof of Theorem 22, save for the last step. Consider first the case $c$ even. Let $\left(Z, T_{1}, T_{2}\right)$ be the telescoping triple corresponding to $(c, \chi)$ as in the first part of the proof of Theorem 22 If $k=0$, then do +1 Luttinger surgery on $T_{1}$ to get a minimal (by Proposition 13 and Usher's theorem) manifold $Z_{1}$ with $\pi_{1}\left(Z_{1}\right) \cong \mathbb{Z}$ containing a symplectic torus $T_{2}$ (after perturbing the symplectic structure) so that the induced map $\pi_{1}\left(T_{2}\right) \rightarrow \pi_{1}\left(Z_{1}\right)$ is a split surjection. If $k \geq 1$, then take a fiber sum of $Z$ with $E(k)$ to again get a manifold $Z_{1}$ with $\pi_{1}\left(Z_{1}\right) \cong \mathbb{Z}$ containing a symplectic torus $T_{2}$ so that the induced map $\pi_{1}\left(T_{2}\right) \rightarrow \pi_{1}\left(Z_{1}\right)$ is a surjection.

Next consider the case of $c$ odd. Following the proof of Theorem 22 let $\left(c^{\prime}, \chi^{\prime}\right)=$ $(c-1, \chi-2)$ and construct the telescoping triple $\left(Z, T_{1}, T_{2}\right)$ corresponding to the pair $\left(c^{\prime}, \chi^{\prime}\right)$. If $k=0$, form the symplectic sum of $Z$ along $T_{1}$ with $S_{1,1}$ and perturb the symplectic form so that $T_{2}$ is symplectic. If $k \geq 1$, form the symplectic sum of $Z$ along $T_{1}$ with $E(k)$ along an elliptic fiber, then form a further symplectic sum along a different elliptic fiber of $E(k)$ with $S_{1,1}$. Then perturb the symplectic form so that $T_{2}$ becomes symplectic. In either case, this results in a minimal symplectic manifold $Z_{1}$ with $\pi_{1}\left(Z_{1}\right) \cong \mathbb{Z}$ containing a symplectic torus $T_{2}$ so that the induced map $\pi_{1}\left(T_{2}\right) \rightarrow \pi_{1}\left(Z_{1}\right)$ is a surjection and $\left(c_{1}^{2}, \chi_{h}\right)=(c, \chi)$.

Since the meridian of $T_{2}$ is nullhomotopic in $Z_{1}$, the symplectic sum, $Y$, of $Z_{1}$ and $X$ has fundamental group isomorphic to that of $X$, since the homomorphism $\pi_{1}(T) \rightarrow$ $\pi_{1}(X)$ is trivial. Minimality follows as in the proof of Theorem 22 using Usher's theorem. Since $c_{1}^{2}$ and $\chi_{h}$ are both additive with respect to symplectic sums along tori, the result follows.

Before we can prove Theorem B stated in the introduction, we will require one more useful fact about $B$ and $X_{1,3}$ not mentioned in Theorem 7 or Corollary 8 , namely, the existence of a genus 2 square zero symplectic surface $G$ geometrically dual to $F$. We indicate how to find $G: X_{1,3}$ is obtained by Luttinger surgeries on eight Lagrangian tori in the symplectic sum of the twice blown up 4-torus $\left(T^{2} \times T^{2}\right) \# 2 \overline{\mathbb{C P}}^{2}$ and the product $T \times F_{2}$ of a torus and a genus 2 surface.

This symplectic sum is taken along the genus 2 surface in $\left(T^{2} \times T^{2}\right) \# 2 \overline{\mathbb{C P}}^{2}$ obtained by resolving $\left(T^{2} \times\{p\}\right) \cup\left(\{q\} \times T^{2}\right)$ and blowing up twice (for definiteness at points on $\left.T^{2} \times\{p\}\right)$. In $T \times F_{2}$ one takes the surface $\{x\} \times F_{2}$.

The square -1 torus $H_{1}$ of Theorem 7 and Corollary 8 was obtained by taking the torus of the form $T \times\{z\}$ which matches up with one of the exceptional spheres in the symplectic sum. To find the surface $G$, take another nearby torus of the form $T \times\left\{z^{\prime}\right\}$ in $T \times F_{2}$ and match it up with a torus of the form $\left\{q^{\prime}\right\} \times T^{2}$. This is the required surface $G$. (The surface $F$ is a parallel copy of $\{x\} \times F_{2}$.) 
Proof of Theorem $B$. Start with the telescoping triple $\left(B, T_{1}, T_{2}\right)$ of Theorem 7 . It contains a genus 2 square zero symplectic surface $F$ and a geometrically dual square zero symplectic genus 2 surface $G$. The union $F \cup G$ is disjoint from $T_{1} \cup T_{2}$.

Perform +1 Luttinger surgery on $T_{1}$ along $\ell_{T_{1}}$ to kill $t_{2}$. Call the result $R$. Perturb the symplectic form on $R$ slightly so that $T_{2}$ becomes symplectic. Note that $\pi_{1}\left(R-T_{2}\right)=$ $\pi_{1}(R)=\mathbb{Z} t_{1}, \pi_{1}\left(T_{2}\right) \rightarrow \pi_{1}(R)$ is surjective, and $R$ is minimal (Proposition 13).

In [6, Theorem 18], a minimal symplectic 4-manifold $\tilde{X}_{3,5}$ homeomorphic to $3 \mathbb{C P}^{2}$ \# $5 \overline{\mathbb{C P}}^{2}$ and containing a pair of symplectic tori $T_{3}, T_{4}$ with simply connected complement is constructed. The symplectic sum $Q=R \#_{T_{2}, T_{3}} \tilde{X}_{3,5}$ is minimal by Usher's theorem. Moreover, $Q$ is simply connected, since $T_{2} \subset R$ induces a surjection on fundamental groups. The surfaces $F$ and $G$ persist as square zero, symplectic, geometrically dual surfaces. Since $e(Q)=16$ and $\sigma(Q)=-4, Q$ is neither rational nor ruled. Notice that the symplectic torus $T_{4}$ in $Q$ has simply connected complement.

In $Q$, take eight parallel copies of the genus 2 surface $F$ and one copy of $G$ and symplectically resolve to obtain a genus 18 surface $\Sigma \subset Q$ of square 16 . Blow up $Q 16$ times, yielding a genus 18 square zero surface $\tilde{\Sigma} \subset \tilde{Q}=Q \# 16 \overline{\mathbb{C P}}^{2}$. By Li's theorem, every -1 sphere in $\tilde{Q}$ intersects $\tilde{\Sigma}$. Moreover, $\pi_{1}(\tilde{Q}-\tilde{\Sigma})=1$.

In [32, Lemma 2.1], a Lefschetz fibration $H \rightarrow K$ over a surface $K$ of genus 2 is constructed which has $e=75$ and $\sigma=25$. This fibration admits a symplectic section of square -1 and has fiber genus 16. The 4-manifold $H$ is an algebraic surface, and by the Bogomolov-Miyaoka-Yau inequality [7] is holomorphically minimal. By [18], it is also symplectically minimal. Moreover, $H$ is neither rational nor ruled since it lies on the BMY line.

Let $\Sigma^{\prime} \subset H$ denote the symplectic surface obtained by symplectically resolving the union of a fiber and a section. Then $\Sigma^{\prime}$ has square 1 , and the exact sequence of fundamental groups for a Lefschetz fibration shows that $\pi_{1}\left(\Sigma^{\prime}\right) \rightarrow \pi_{1}(H)$ is surjective. Blow up $H$ once along $\Sigma^{\prime}$ and take the proper transform to obtain a square zero, genus 18 surface $\tilde{\Sigma}^{\prime} \subset \tilde{H}=H \# \overline{\mathbb{C P}}^{2}$ so that $\pi_{1}\left(\tilde{H}-\tilde{\Sigma}^{\prime}\right) \rightarrow \pi_{1}(\tilde{H})$ is an isomorphism and $\pi_{1}\left(\tilde{\Sigma}^{\prime}\right) \rightarrow \pi_{1}(\tilde{H})$ is surjective. By Li's theorem (Theorem 6), every -1 sphere in $\tilde{H}$ intersects $\tilde{\Sigma}^{\prime}$, since $H$ is neither rational nor ruled.

Hence the symplectic sum $S=\tilde{Q} \#_{\tilde{\Sigma}, \tilde{\Sigma}^{\prime}} \tilde{H}$ is minimal. It is simply connected since $\pi_{1}(\tilde{Q}-\tilde{\Sigma})=1$ and $\pi_{1}\left(\tilde{\Sigma}^{\prime}\right) \rightarrow \pi_{1}(\tilde{H})$ is surjective. Moreover, the symplectic torus $T_{4} \subset S$ has simply connected complement.

Since $S$ is the symplectic sum along genus 18 surfaces,

$$
\begin{aligned}
e(S) & =e(\tilde{Q})+e(\tilde{H})+4(18-1)=176, \\
\sigma(S) & =\sigma(\tilde{Q})+\sigma(\tilde{H})=24-20=4 .
\end{aligned}
$$

Thus $c_{1}^{2}(S)=364$ and $\chi_{h}(S)=45$. It contains the symplectic torus $T_{4}$ with simply connected complement. Hence Theorem 23 establishes the existence of minimal, simply connected symplectic 4-manifolds

$$
X_{89+2 \chi, 85+10 \chi-c}
$$


with $c_{1}^{2}=364+c$ and $\chi_{h}=45+\chi$ for any $(c, \chi)$ satisfying $0 \leq c \leq 8 \chi-2$ when $c$ is even.

Taking $c=8 \chi-4$ for any $\chi \geq 1$ yields $X_{89+2 \chi}, 89+2 \chi$, a minimal simply connected symplectic 4-manifold with signature zero. The intersection form is odd since, as one can check from Lemma 21, either $\chi=1$ in which case the model manifold $C$ (with its -1 torus) is used in the construction of $X_{91,91}$, or else $\chi>1$, in which case the model manifold $B$ (with its -1 torus) is used in the construction of $X_{89+2 \chi}, 89+2 \chi$.

To get minimal symplectic 4-manifolds with signature -1 , consider the symplectic sum

$$
Y=B \#_{T_{1}, T} P_{1+2 k, 4+2 k}
$$

of the manifold $B$ of Theorem 7 with the manifold $P_{1+2 k, 4+2 k}$ of Remark 1 (at the end of Section 6 along $T_{1}$ in $B$ and $T$ in $P_{1+2 k, 4+2 k}$. Since $\pi_{1}(T) \rightarrow \pi_{1}\left(P_{1+2 k, 4+2 k}\right)=\mathbb{Z}$ is surjective, $\pi_{1}\left(P_{1+2 k, 4+2 k}-T\right) \rightarrow \pi_{1}\left(P_{1+2 k, 4+2 k}\right)$ is an isomorphism, and $\pi_{1}\left(T_{1}\right) \rightarrow$ $\pi_{1}(B)$ has image a cyclic summand, the gluing map for the symplectic sum can be chosen so that $B-\operatorname{nbd}\left(T_{1}\right) \subset Y$ induces an isomorphism on fundamental groups. Hence $\pi_{1}\left(T_{2}\right) \rightarrow \pi_{1}(Y)$ is an isomorphism.

The symplectic sum

$$
X_{93+2 k, 94+2 k}=Y \#_{T_{2}, T_{4}} S
$$

is a simply connected minimal symplectic 4-manifold with $e=189+4 k$ and $\sigma=-1$, for any $k \geq 2$.

Since any symplectic signature zero 4-manifold has $e$ a multiple of 4 , there remain 45 signature zero minimal symplectic 4-manifolds with odd intersection form to be constructed. Also missing are 48 signature -1 minimal symplectic 4 -manifolds. Hence to complete the geography problem for minimal simply connected symplectic 4-manifolds of non-positive signature and odd intersection form, there remain 97 manifolds to discover.

Acknowledgments. The authors would like to thank R. E. Gompf for helpful comments.

A. A. was partially supported by the NSF grant FRG-0244663. S. B. was partially supported by the NSF grant DMS-0507857. R. İ. B. was partially supported by the NSF Grant DMS-0305818. P. K. was partially supported by the NSF grant DMS-0604310. B. D. P. was partially supported by an NSERC discovery grant.

\section{References}

[1] Auroux, D., Donaldson, S. K., Katzarkov, L.: Luttinger surgery along Lagrangian tori and non-isotopy for singular symplectic plane curves. Math. Ann. 326, 185-203 (2003) Zbl 1026.57020 MR 1981618

[2] Akhmedov, A.: Small exotic 4-manifolds. Algebr. Geom. Topology 8, 1781-1794 (2008) MR 2448872

[3] Akhmedov, A., Baykur, R. İ., Park, B. D.: Constructing infinitely many smooth structures on small 4-manifolds. J. Topology 1, 409-428 (2008) Zbl 1146.57041 MR 2399137

[4] Akhmedov, A., Park, B. D.: Exotic smooth structures on small 4-manifolds. Invent. Math. 173, 209-223 (2008) Zbl 1144.57026 MR 2403396 
[5] Baldridge, S., Kirk, P.: A symplectic manifold homeomorphic but not diffeomorphic to $\mathbb{C P}^{2} \# 3 \overline{\mathbb{C P}}^{2}$. Geom. Topology 12, 919-940 (2008) Zbl 1152.57026 MR 2403801

[6] Baldridge, S., Kirk, P.: Constructions of small symplectic 4-manifolds using Luttinger surgery. J. Differential Geom. 82, 317-361 (2009) MR 2520795

[7] Barth, W., Peters, C., Van de Ven, A.: Compact Complex Surfaces. Ergeb. Math. Grenzgeb. (3) 4, Springer, Berlin (1984) Zbl 0718.14023 MR 0749574

[8] Fintushel, R., Park, B. D., Stern, R. J.: Reverse engineering small 4-manifolds. Algebr. Geom. Topology 7, 2103-2116 (2007) Zbl 1142.57018 MR 2366188

[9] Fintushel, R., Stern, R. J.: Surgery in cusp neighborhoods and the geography of irreducible 4-manifolds. Invent. Math. 117, 455-523 (1994) Zbl 0843.57021 MR 1283727

[10] Fintushel, R., Stern, R. J.: Knots, links, and 4-manifolds. Invent. Math. 134, 363-400 (1998) Zbl 0914.57015 MR 1650308

[11] Fintushel, R., Stern, R. J.: Double node neighborhoods and families of simply connected 4-manifolds with $b^{+}=1$. J. Amer. Math. Soc. 19, 171-180 (2006) Zbl 1087.57019 MR 2169045

[12] Fintushel, R., Stern, R. J.: Six lectures on four 4-manifolds. In: Low Dimensional Topology, IAS/Park City Math. Ser. 15, Amer. Math. Soc., 265-315 (2009) MR 2503498

[13] Freedman, M. H.: The topology of four-dimensional manifolds. J. Differential Geom. 17, 357-453 (1982) Zbl 0528.57011 MR 0679066

[14] Friedman, R., Morgan, J. W.: Smooth Four-Manifolds and Complex Surfaces. Ergeb. Math. Grenzgeb. (3) 27, Springer, Berlin (1994) Zbl 0817.14017 MR 1288304

[15] Gompf, R. E.: A new construction of symplectic manifolds. Ann. of Math. (2) 142, 527-595 (1995) Zbl 0849.53027 MR 1356781

[16] Gompf, R. E., Mrowka, T. S.: Irreducible 4-manifolds need not be complex. Ann. of Math. (2) 138, 61-111 (1993) Zbl 0805.57012 MR 1230927

[17] Gompf, R. E., Stipsicz, A. I.: 4-manifolds and Kirby Calculus. Grad. Stud. Math. 20, Amer. Math. Soc., Providence, RI (1999) Zbl 0933.57020 MR 1707327

[18] Hamilton, M. J. D., Kotschick, D.: Minimality and irreducibility of symplectic four-manifolds. Int. Math. Res. Notices 2006, art. ID 35032, 13 pp. Zbl 1101.53052 MR 2211144

[19] Kotschick, D.: On manifolds homeomorphic to $\mathbb{C P}^{2} \# 8 \overline{\mathbb{C P}}^{2}$. Invent. Math. 95, 591-600 (1989) Zbl 0691.57008 MR 0979367

[20] Li, T.-J.: Smoothly embedded spheres in symplectic 4-manifolds. Proc. Amer. Math. Soc. 127, 609-613 (1999) Zbl 0911.57018 MR 1459135

[21] Li, T.-J., Liu, A.: Symplectic structure on ruled surfaces and a generalized adjunction formula. Math. Res. Lett. 2, 453-471 (1995) Zbl 0855.53019 MR 1355707

[22] Luttinger, K. M.: Lagrangian tori in $\mathbb{R}^{4}$. J. Differential Geom. 42, 220-228 (1995) Zbl 0861.53029 MR 1366546

[23] McDuff, D., Salamon, D.: Introduction to Symplectic Topology. 2nd ed., Oxford Math. Monogr., Oxford Univ. Press, New York (1998) Zbl 1066.53137| MR 1698616

[24] Morgan, J. W., Mrowka, T. S., Szabó, Z.: Product formulas along $T^{3}$ for Seiberg-Witten invariants. Math. Res. Lett. 4, 915-929 (1997) Zbl 0892.57021 MR 1492130

[25] Park, B. D.: Constructing infinitely many smooth structures on $3 \mathbb{C P}^{2} \# n \overline{\mathbb{C P}}^{2}$. Math. Ann. 322, 267-278 (2002); Erratum, Math. Ann. 340, 731-732 (2008) Zbl 0997.57042 MR 2372734

[26] Park, J.: The geography of irreducible 4-manifolds. Proc. Amer. Math. Soc. 126, 2493-2503 (1998) Zbl 0895.57005 MR 1487335

[27] Park, J.: Exotic smooth structures on 4-manifolds. Forum Math. 14, 915-929 (2002) Zbl 1009.57029 MR 1932526 
[28] Park, J.: Simply connected symplectic 4-manifolds with $b_{2}^{+}=1$ and $c_{1}^{2}=2$. Invent. Math. 159, 657-667 (2005) Zbl 1080.57030 MR 2125736

[29] Park, J.: Exotic smooth structures on $3 \mathbb{C P}^{2} \# 8 \overline{\mathbb{C P}}^{2}$. Bull. London Math. Soc. 39, 95-102 (2007) Zbl 1116.57025 MR 2303524

[30] Park, J., Yun, K.-H.: Exotic smooth structures on $(2 n+2 l-1) \mathbb{C P}^{2} \#(2 n+4 l-1) \overline{\mathbb{C P}}^{2}$. Preprint, math.GT/0703619 (2007)

[31] Stipsicz, A. I.: Donaldson invariants of certain symplectic manifolds. J. Reine Angew. Math. 465, 1-10 (1995) Zbl 0828.53027 MR 1344129

[32] Stipsicz, A. I.: Simply connected symplectic 4-manifolds with positive signature. In: Proc. 6th Gökova Geometry-Topology Conference, Turkish J. Math. 23, 145-150 (1999) Zbl 0945.57010 MR 1701643

[33] Stipsicz, A. I., Szabó, Z.: An exotic smooth structure on $\mathbb{C P}^{2} \# 6 \overline{\mathbb{C P}}^{2}$. Geom. Topology 9, 813-832 (2005) Zbl 1077.57025 MR 2140993

[34] Szabó, Z.: Irreducible four-manifolds with small Euler characteristics. Topology 35, 411-426 (1996) Zbl 0874.57022 MR 1380507

[35] Taubes, C. H.: The Seiberg-Witten invariants and symplectic forms. Math. Res. Lett. 1, 809822 (1994) Zbl 0853.57019 MR 1306023

[36] Taubes, C. H.: Counting pseudo-holomorphic submanifolds in dimension 4. J. Differential Geom. 44, 818-893 (1996) Zbl 0883.57020 MR 1438194

[37] Taubes, C. H.: Seiberg-Witten and Gromov invariants. In: Geometry and Physics (Aarhus, 1995), Lecture Notes in Pure Appl. Math. 184, Dekker, New York, 591-601 (1997) Zbl 0873.57017 MR 1423194

[38] Usher, M.: Minimality and symplectic sums. Int. Math. Res. Notices 2006, art. ID 49857, 17 pp. Zbl 1110.57017 MR 2250015 